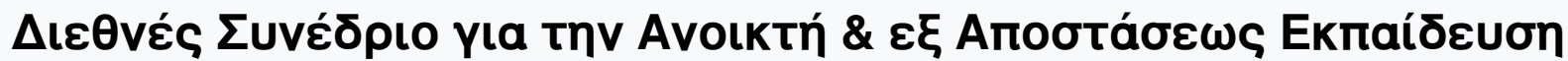

Tón. 6, Ap. 1A (2011)

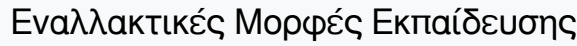

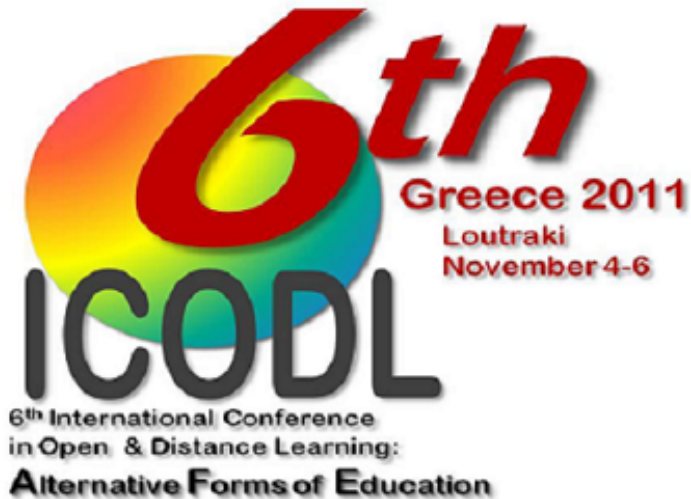

TOMOEA

PART/MEPOEA

in Open \& Distance Learning:

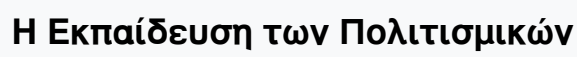

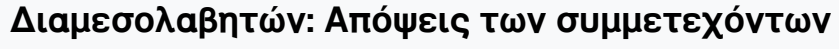

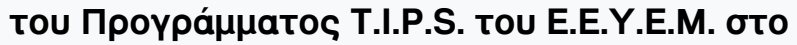

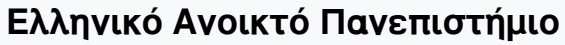

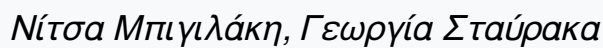

doi: $10.12681 /$ icodl. 682

PART/MEPOEA 


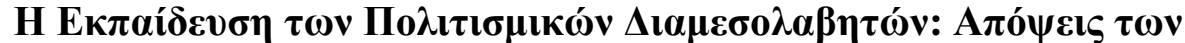

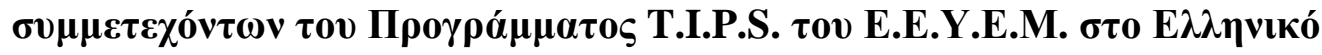

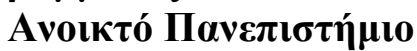

\section{Teaching of cultural mediators: Views of participants to the T.I.P.S. Program of the Laboratory of Educational Material and Methodology at the Hellenic Open University}

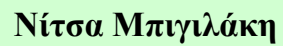

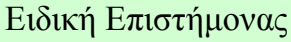

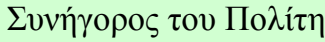

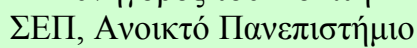 \\ ndimakou@hol.gr
}

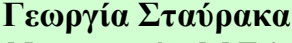

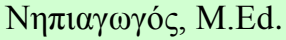 \\ stayrg@yahoo.gr
}

\section{Пєрі́ $\lambda \eta \psi \eta$}

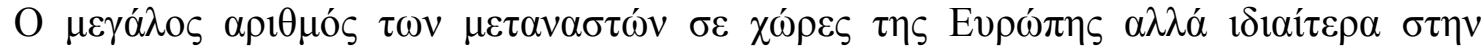

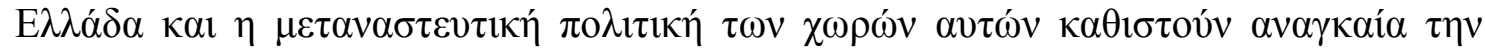

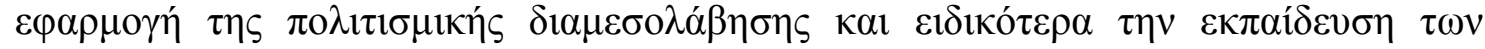

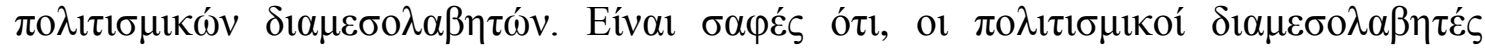

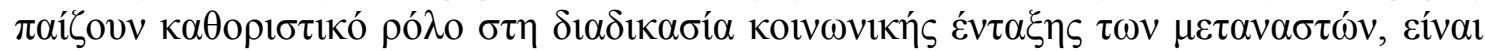

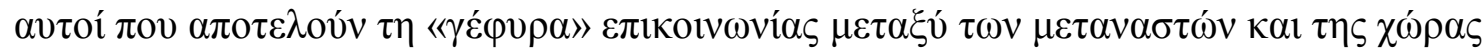

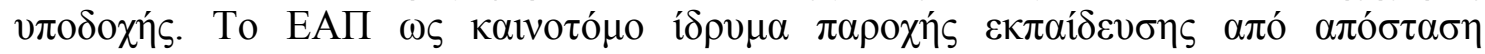

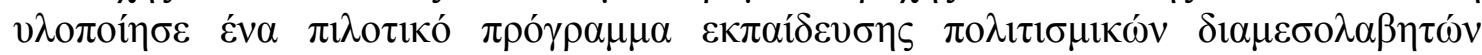

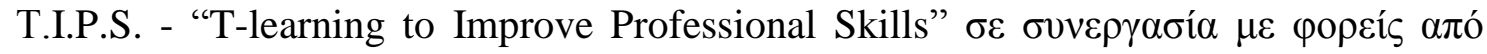
$\alpha \dot{\lambda} \lambda \varepsilon \varsigma \chi \omega \dot{\rho} \rho \varepsilon \varsigma$.

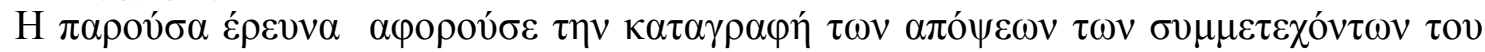

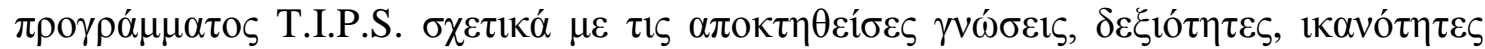

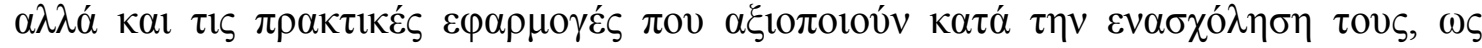

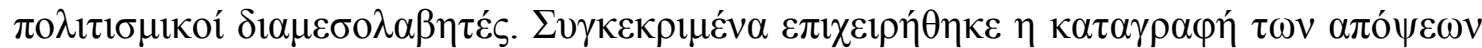

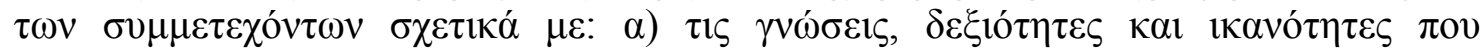

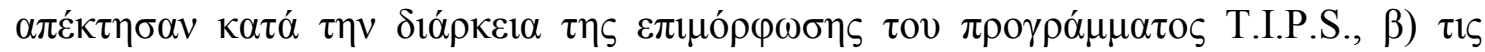

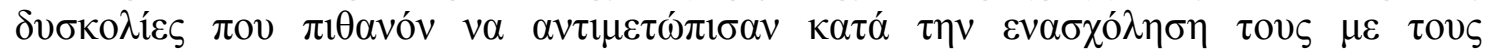

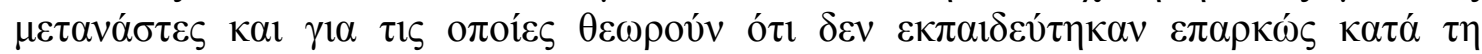

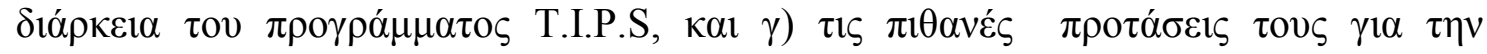

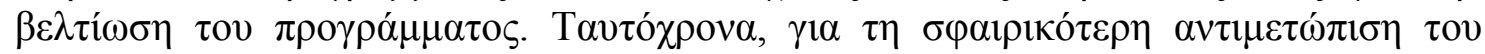

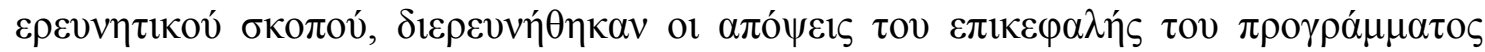

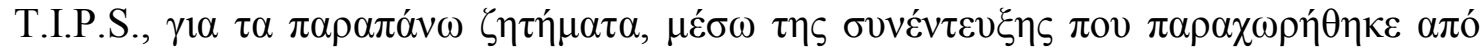

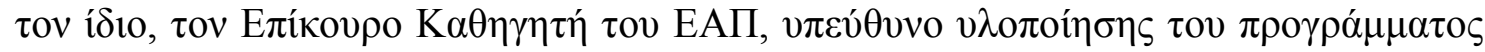

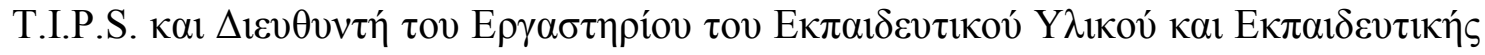

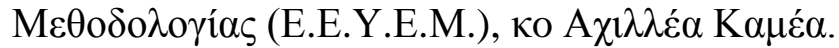

\section{Abstract}

The HELLENIC OPEN UNIVERSITY, as an innovative distance educational institution, it has coordinated a program which was named T.I.P.S. "T-learning to Improve Professional Skills" in order to educate cultural mediators, in collaboration with institutions from other countries. The purpose of this research was to investigate if the participants of the program T.I.P.S. believe that their attendance at the program 
helped them, and to what extent, in their daily practice as cultural mediators. According to their opinions they believe that the program helped them to a large extent and it seems that they have a positive overview of it. Moreover, for a better understanding of the program's implementation, an interview took place with the person in charge of it, the Assistant Professor at the Hellenic Open University, Mr. Achileas Kameas.

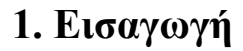

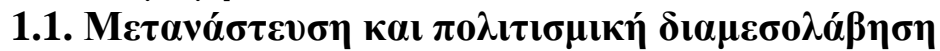

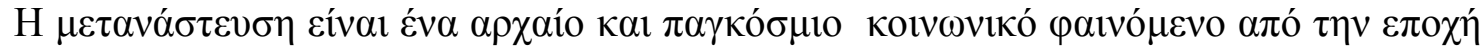

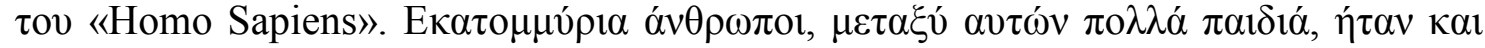

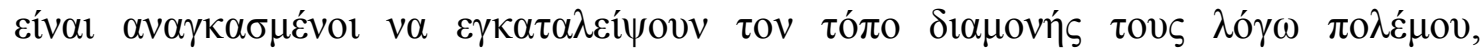

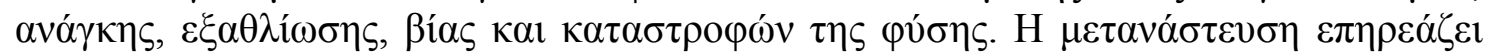

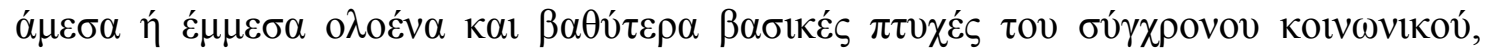

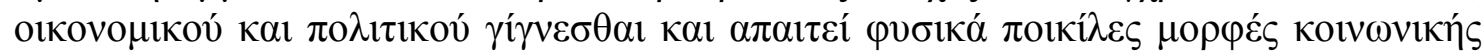

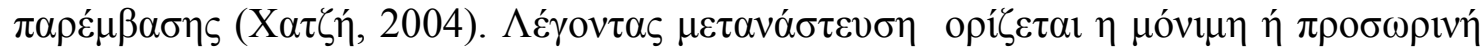

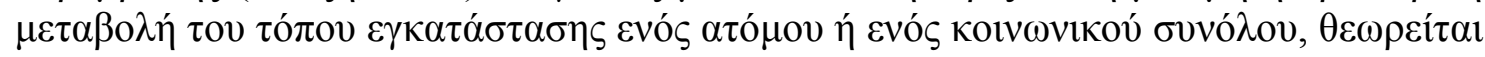

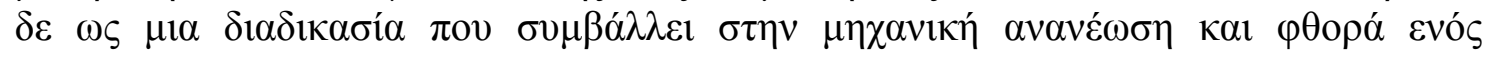

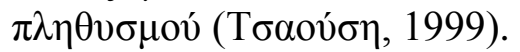

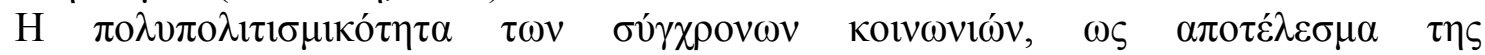

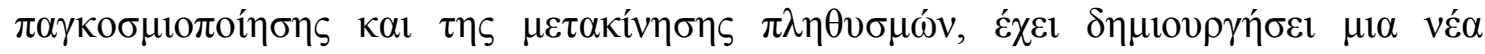

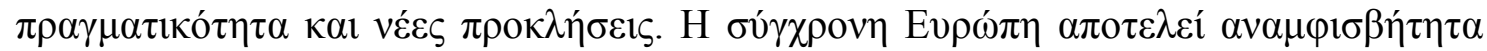

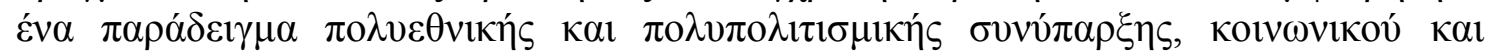

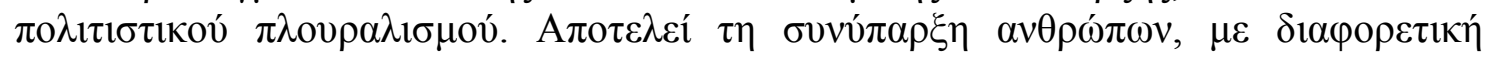

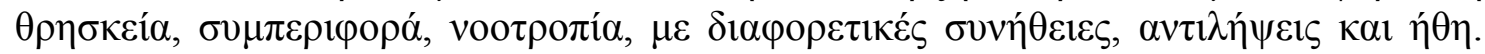

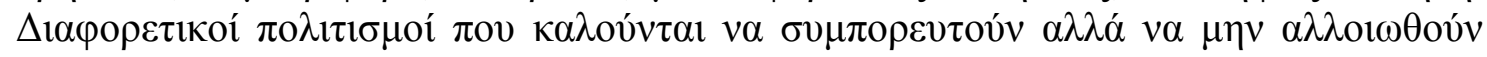

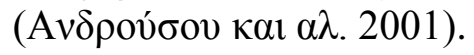

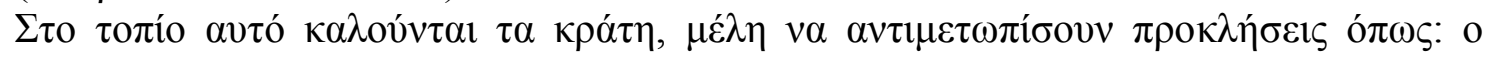

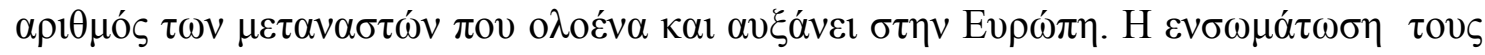

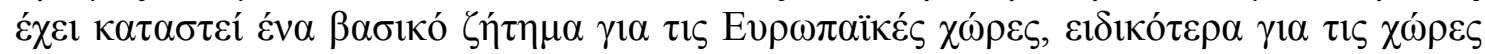

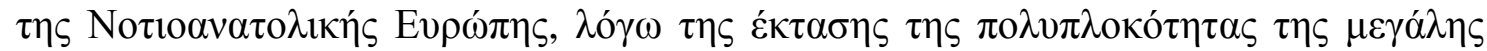

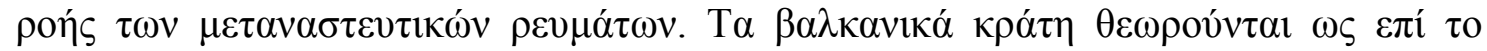

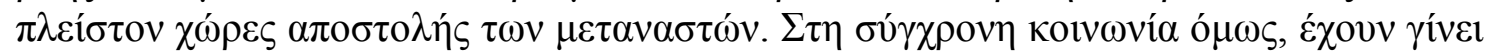

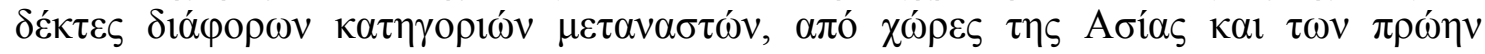

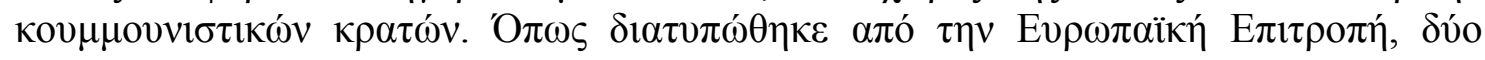

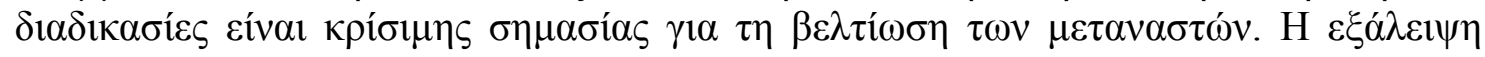

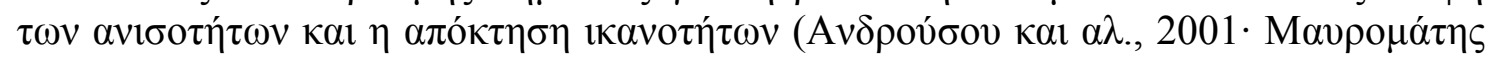

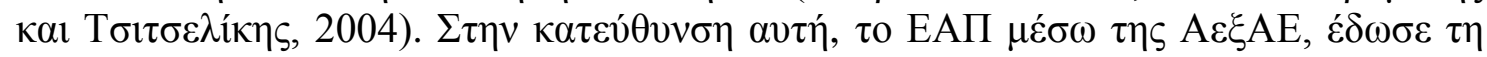

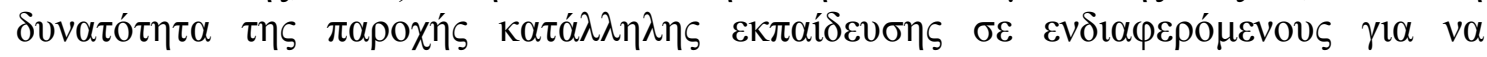

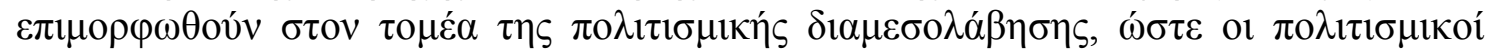

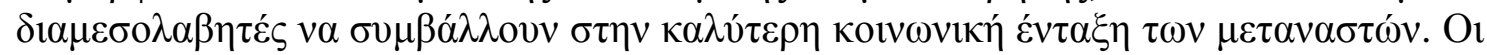

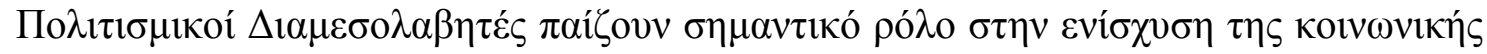

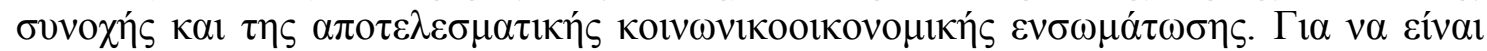

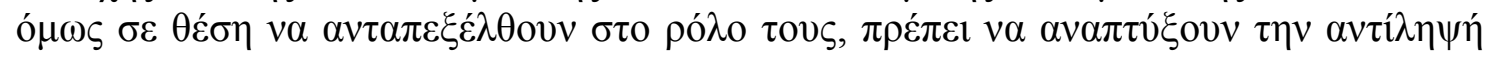

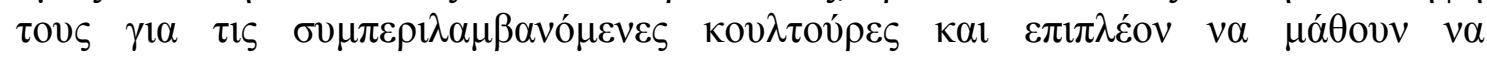

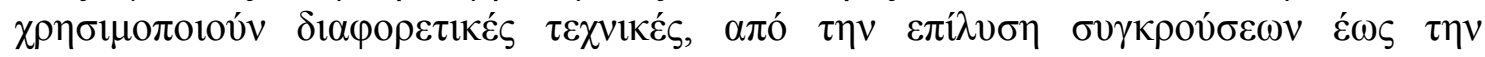

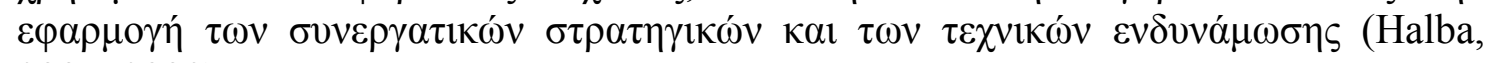
2009, 2008). 


\subsection{Tо $\pi \rho o ́ \gamma \rho \alpha \mu \mu \alpha$ T.I.P.S.}

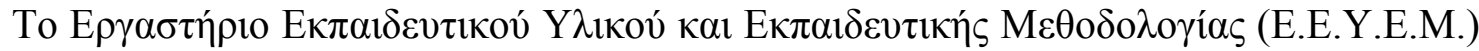

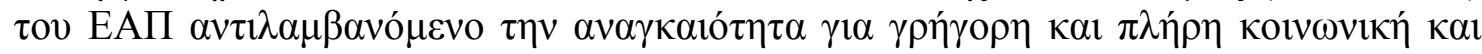

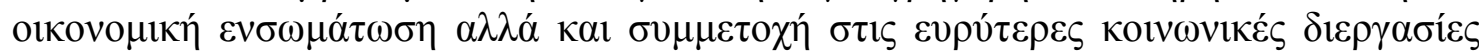

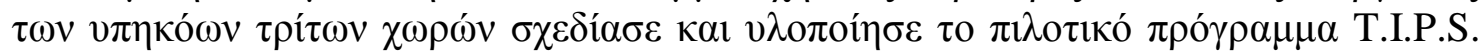

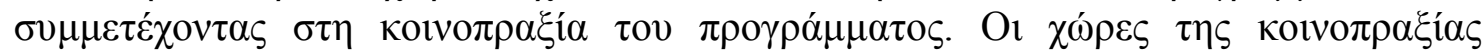

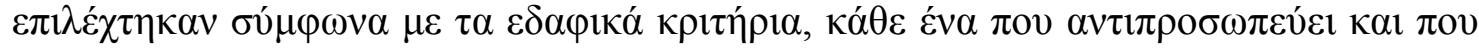

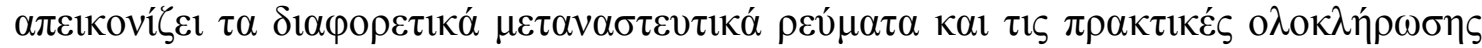

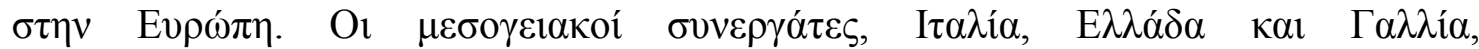

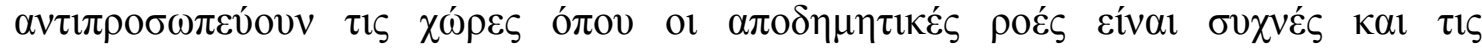

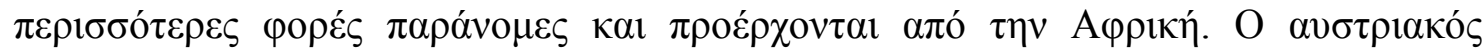

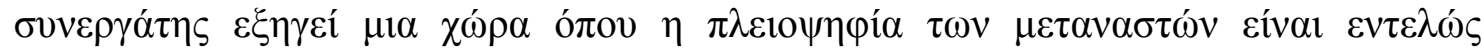

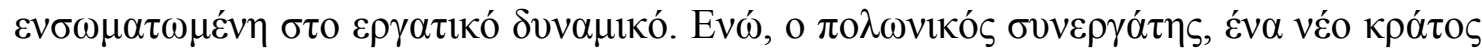

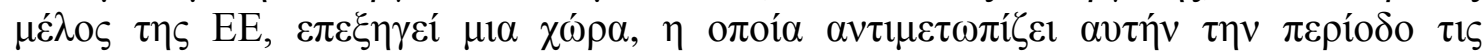

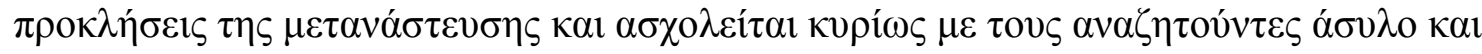

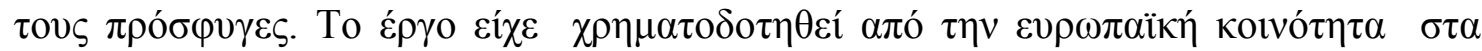

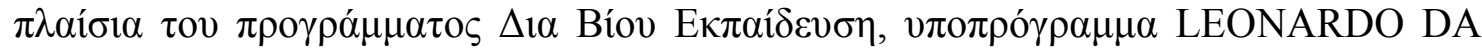

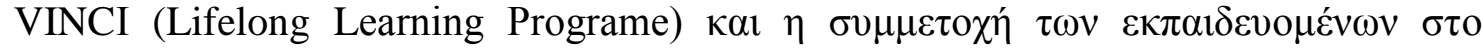

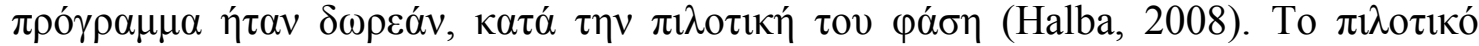

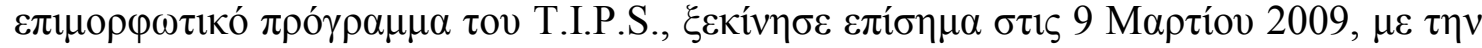

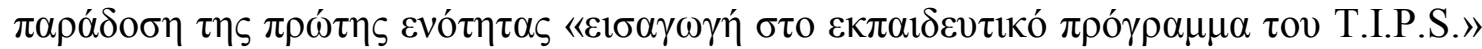

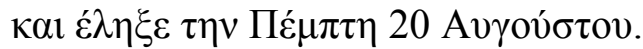

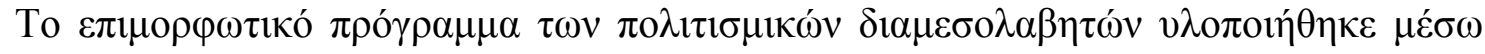

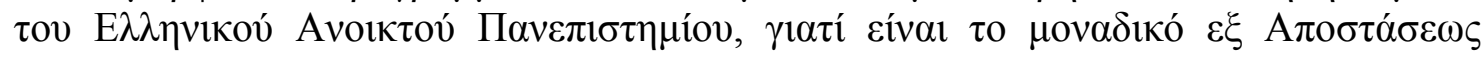

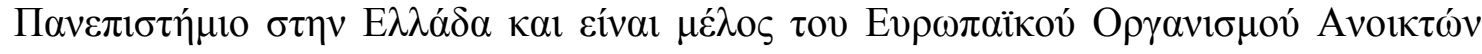

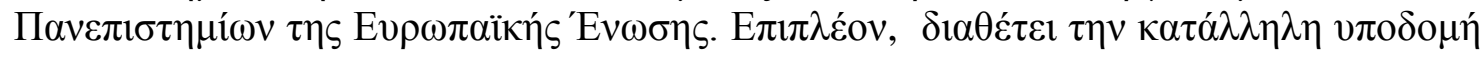

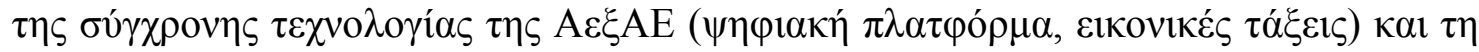

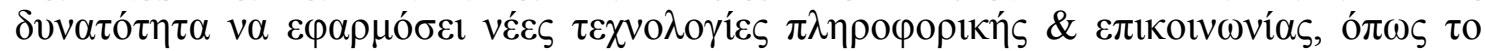

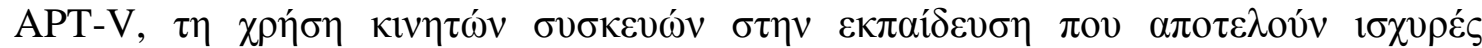

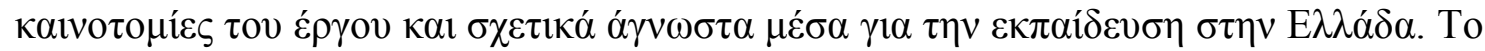

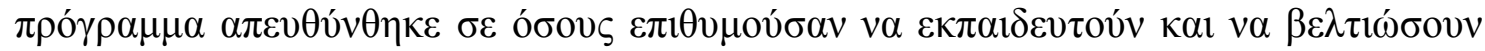

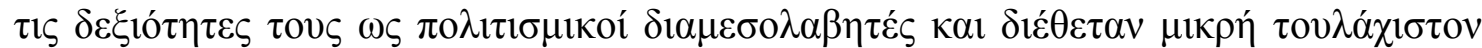

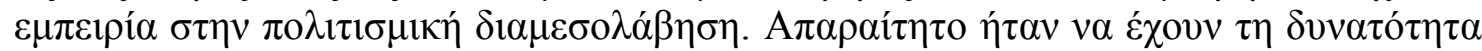

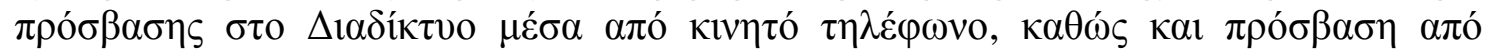

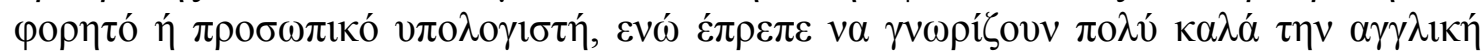

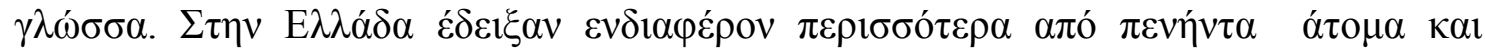

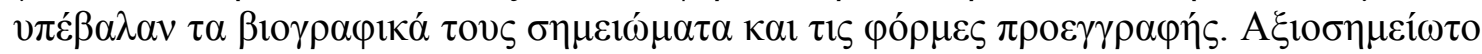

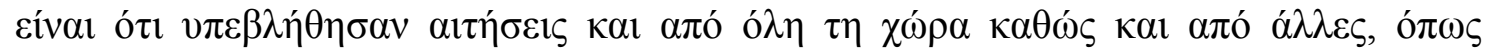

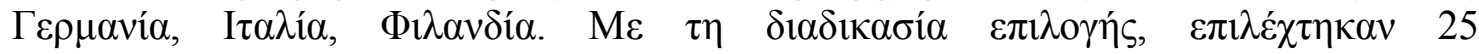

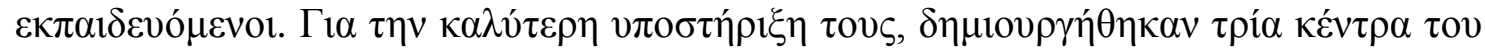

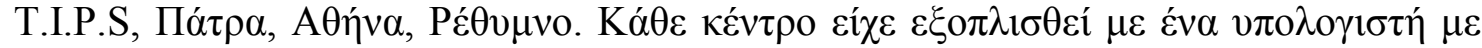

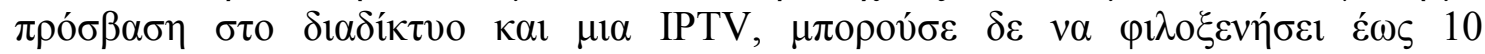

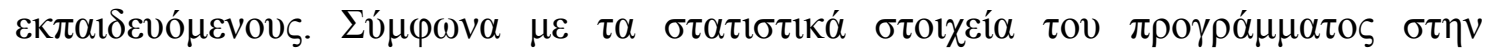

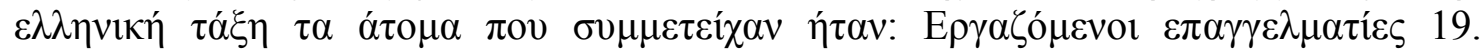

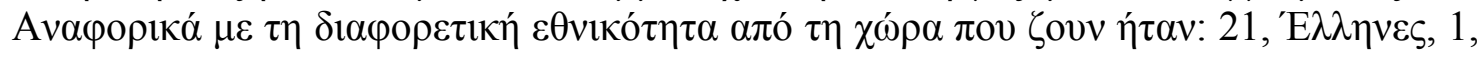

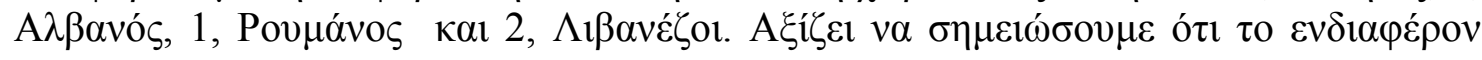

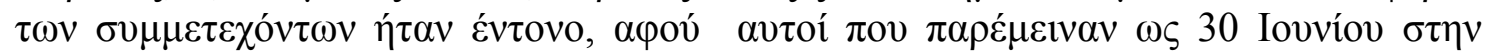

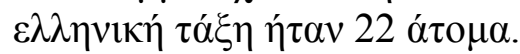

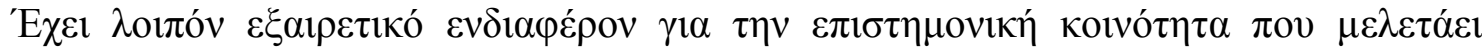

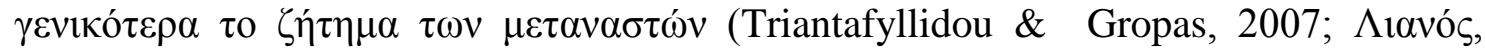




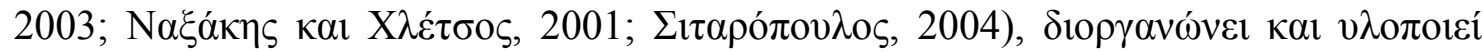

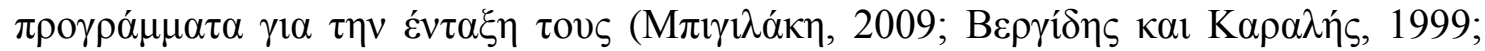

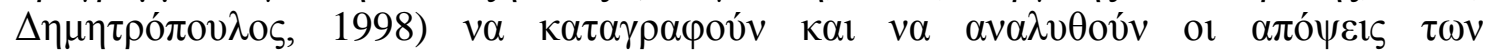

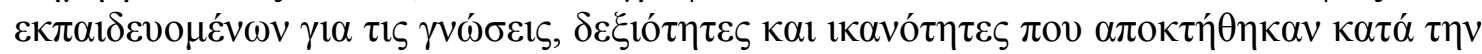

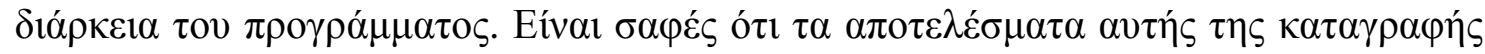

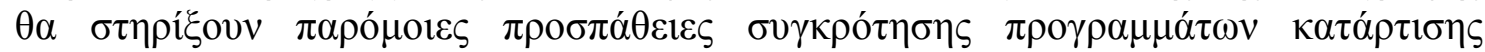

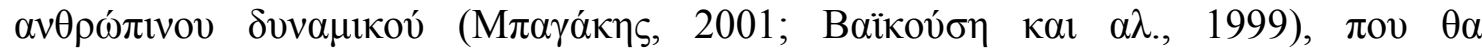

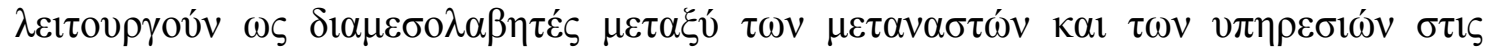

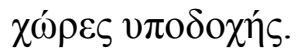

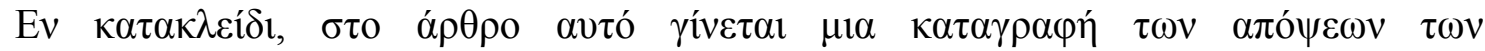

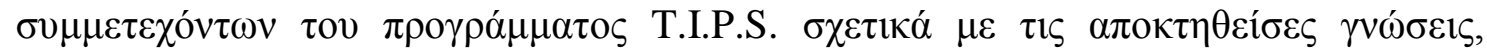

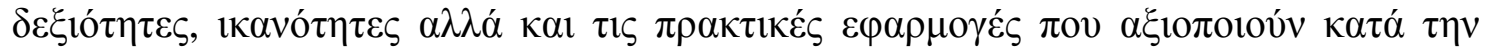

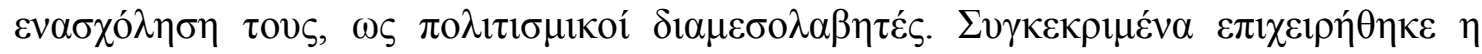

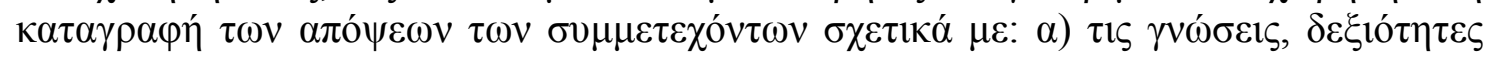

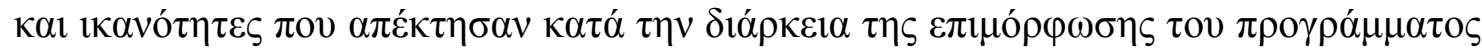

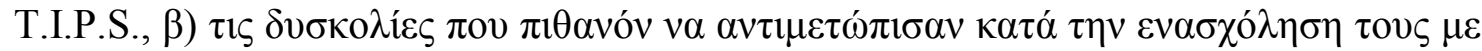

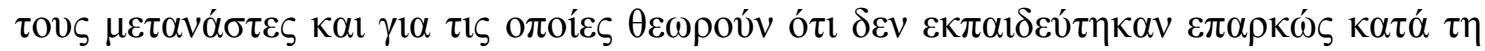

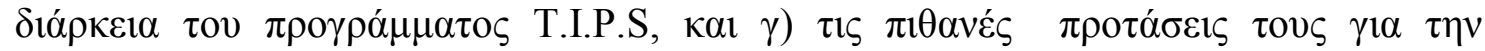

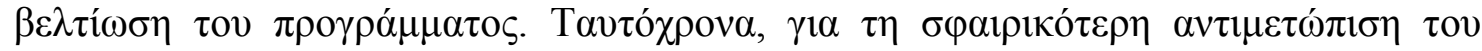

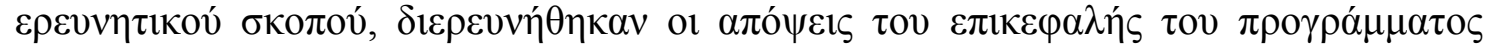

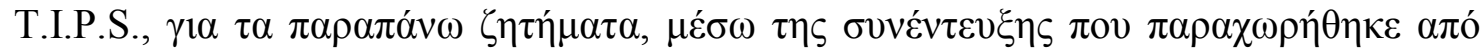

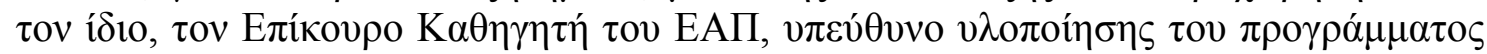

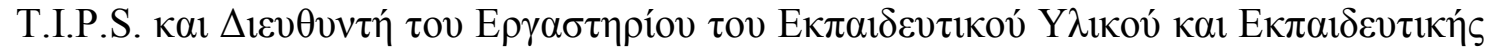

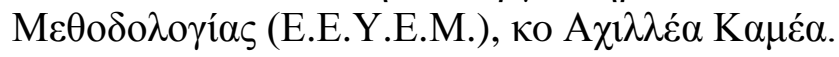

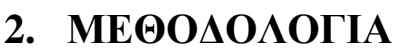

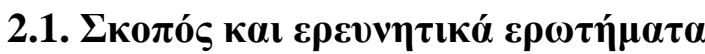

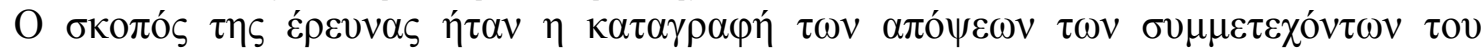

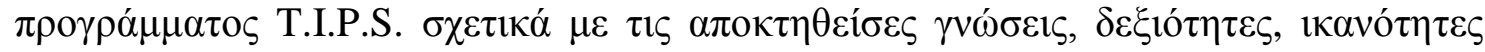

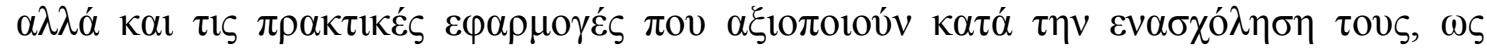

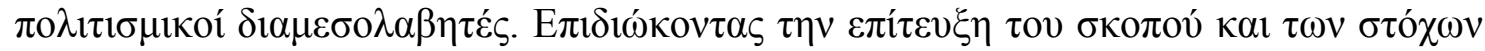

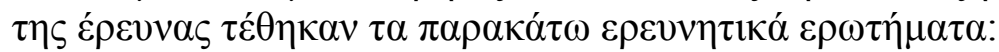

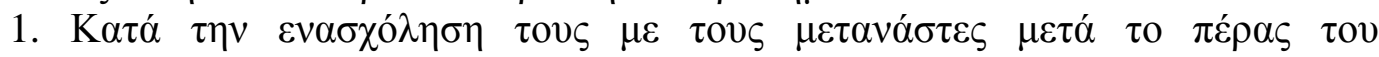

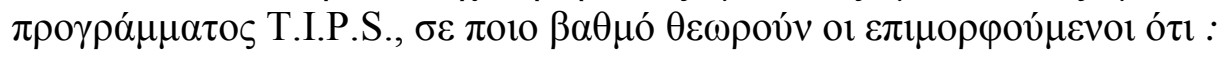

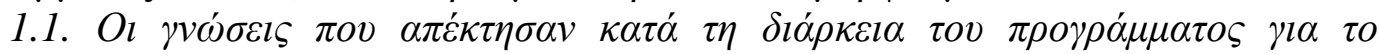

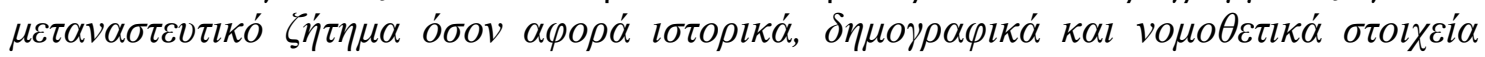

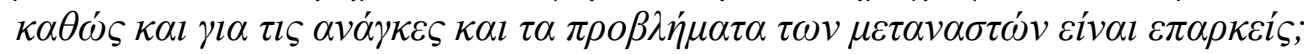

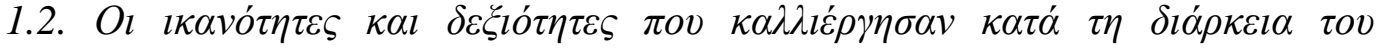

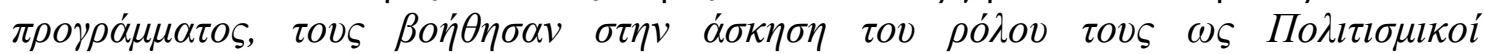
$\Delta \imath \alpha \mu \varepsilon \sigma o \lambda \alpha \beta \eta \tau \dot{\varepsilon} \varsigma$

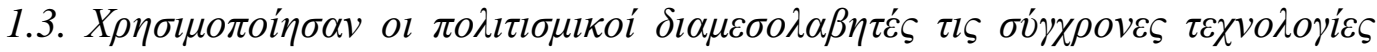

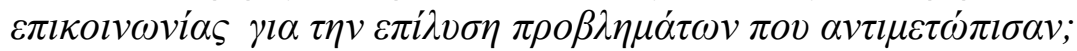

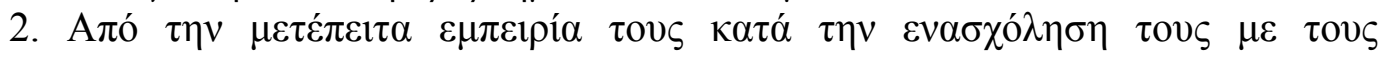

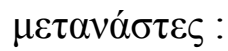

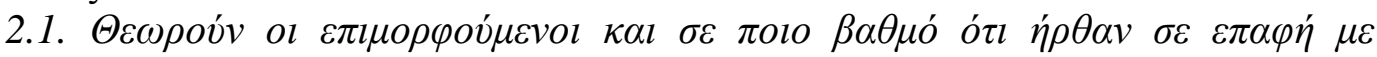

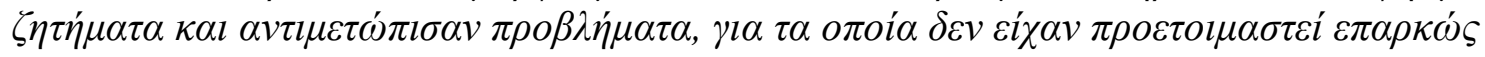

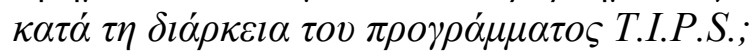

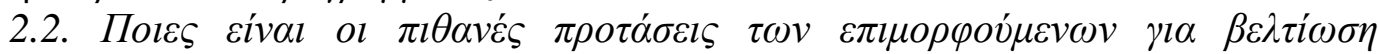

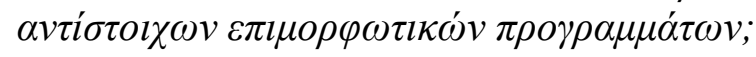




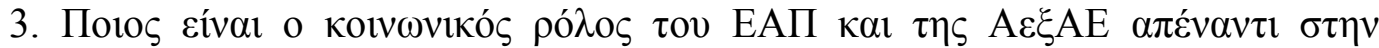

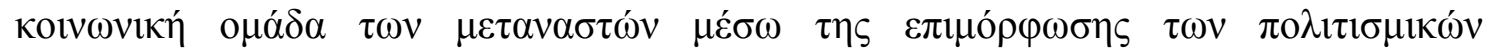
$\delta 1 \alpha \mu \varepsilon \sigma o \lambda \alpha \beta \eta \tau \omega ́ v$

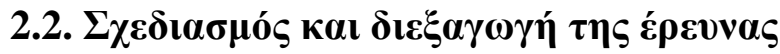

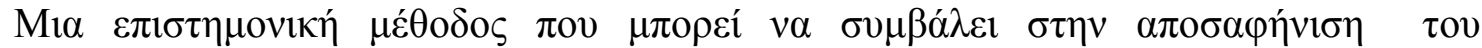

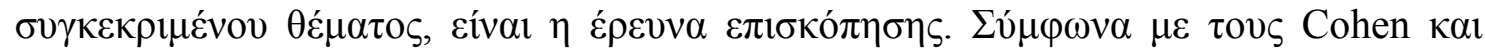

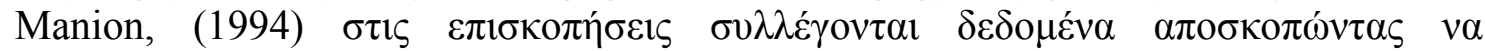

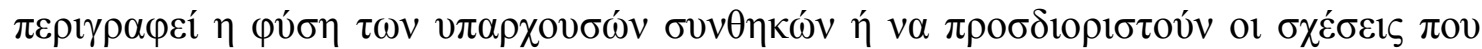

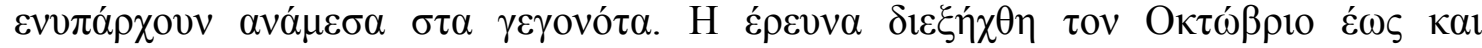

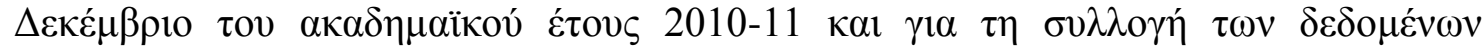

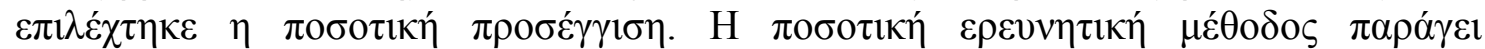

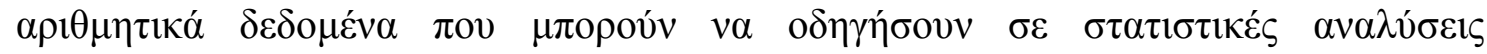

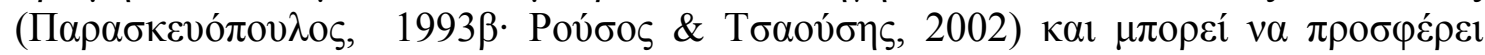

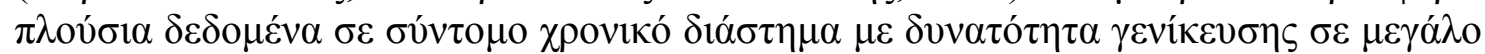

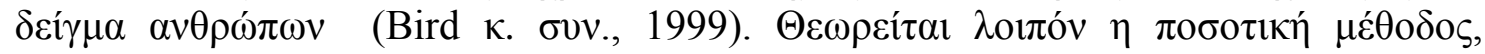

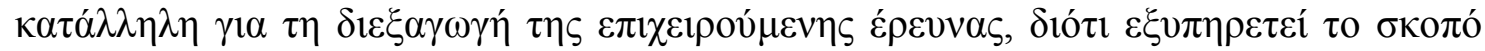

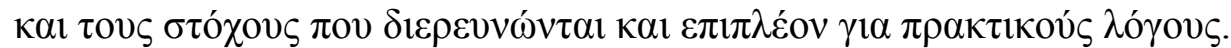

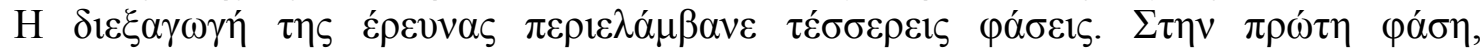

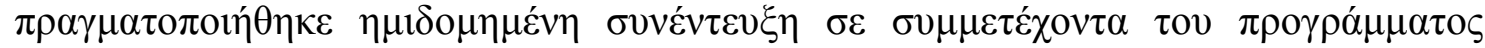

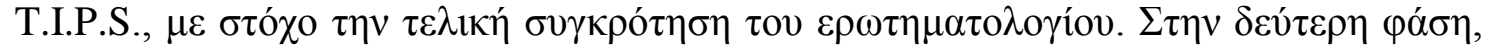

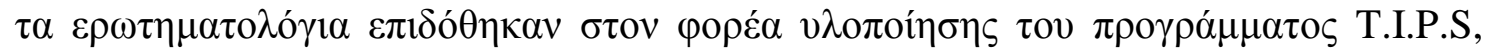

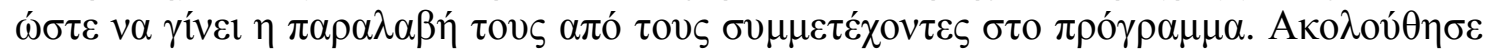

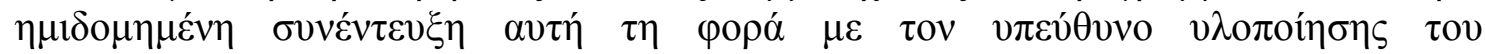

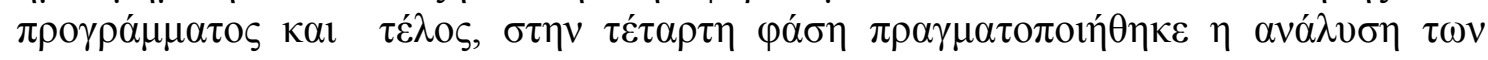

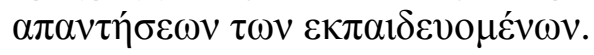

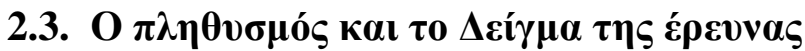

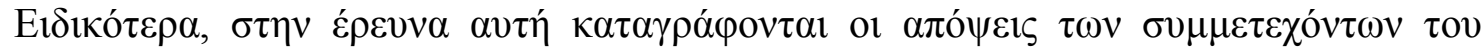

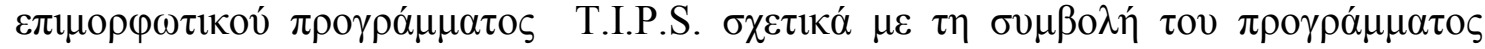

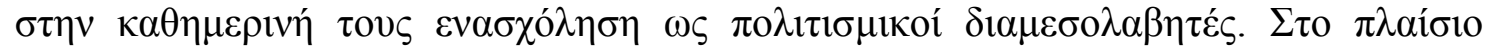

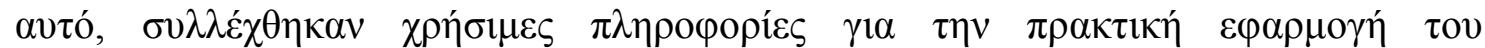

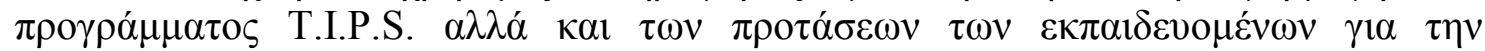

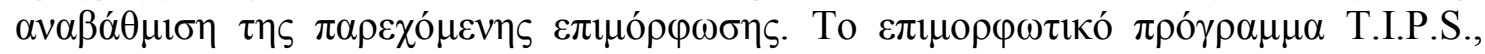

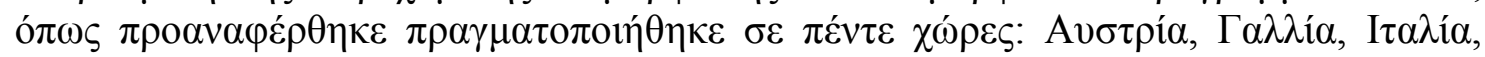

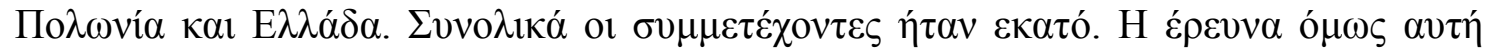

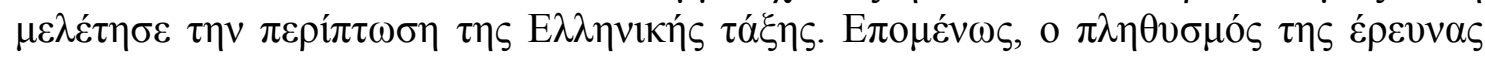

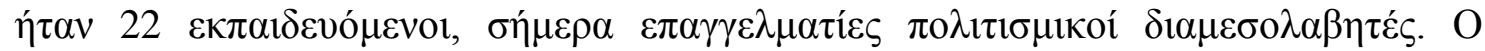

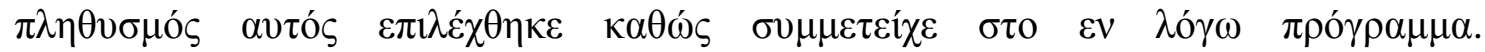

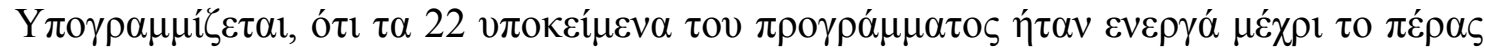

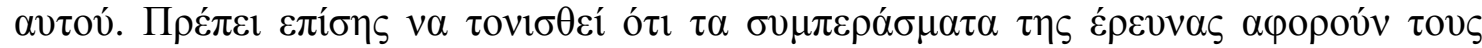

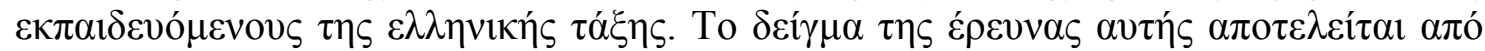

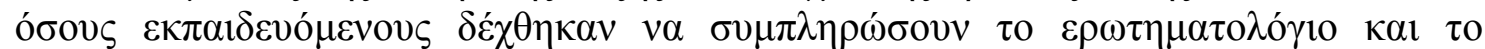

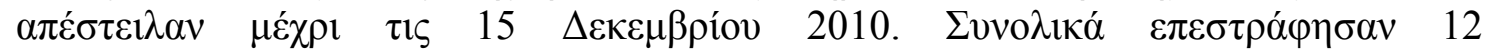

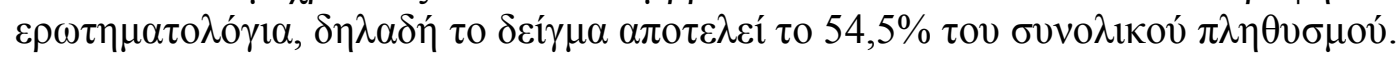

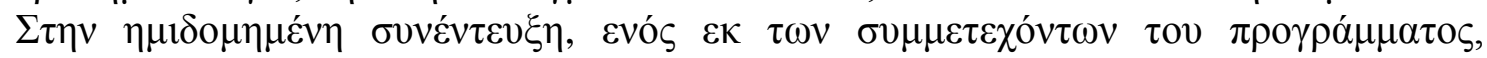

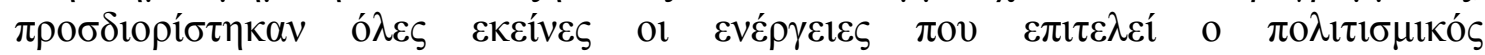

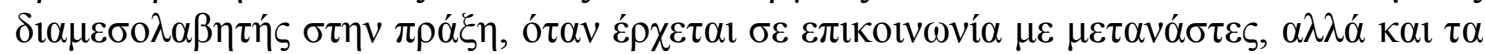

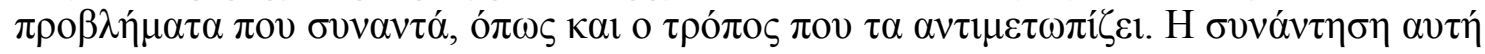




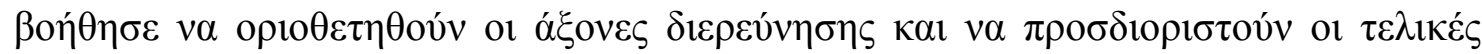

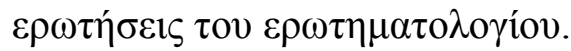

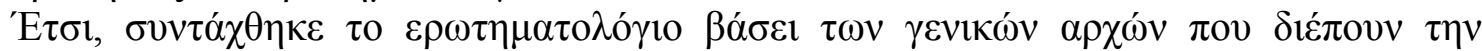

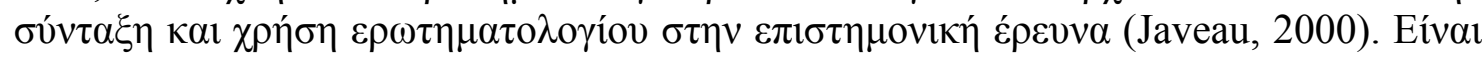

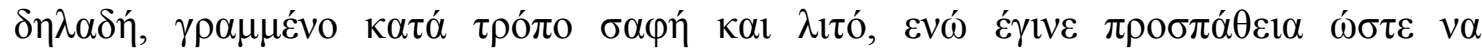

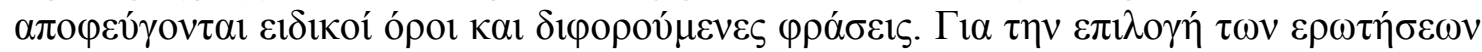

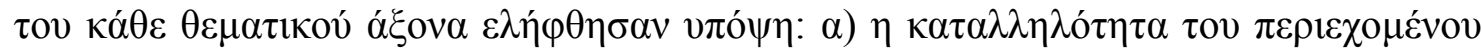

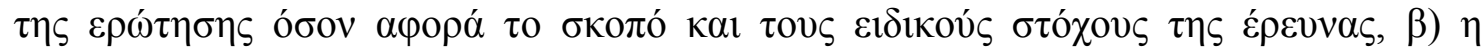

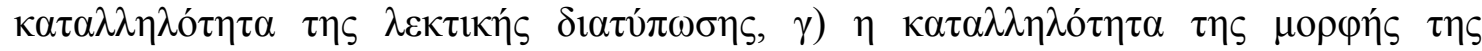

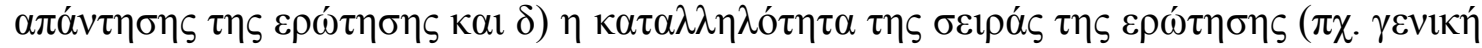

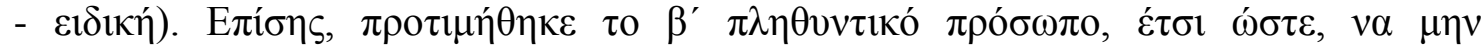

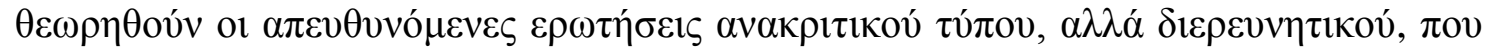

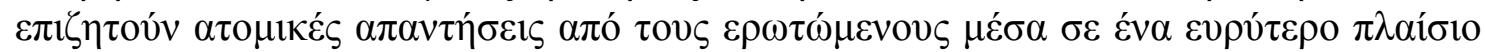

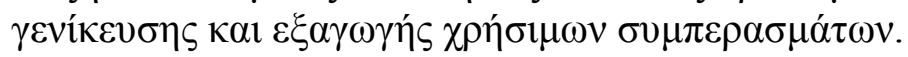

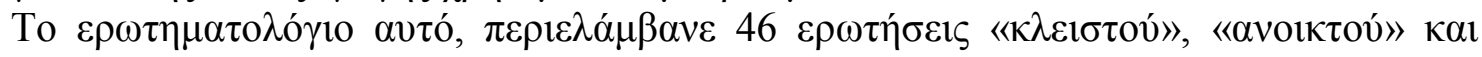

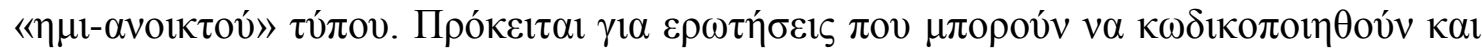

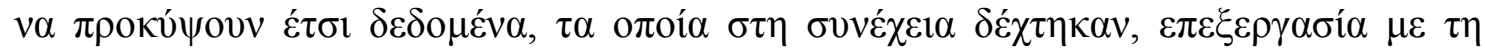

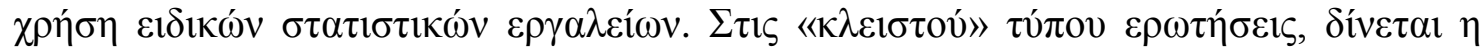

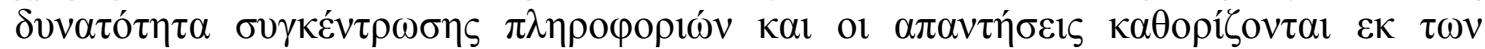

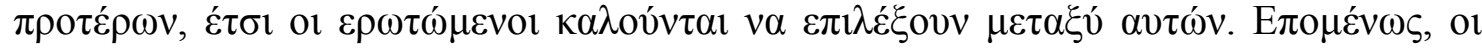

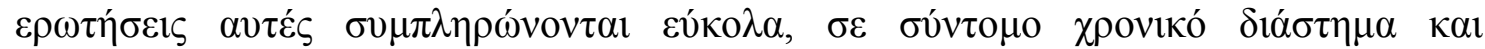

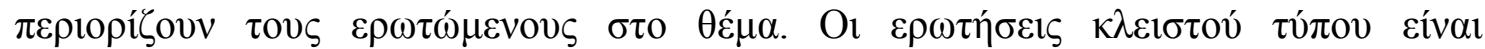

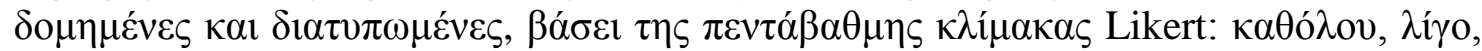

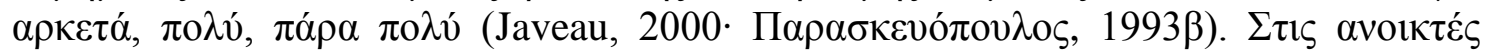

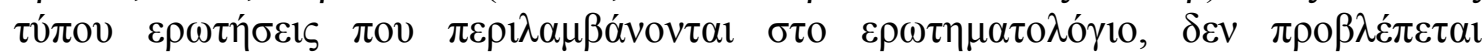

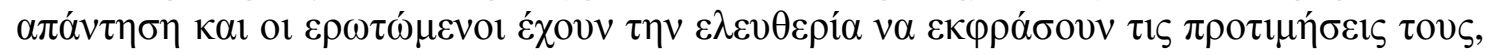

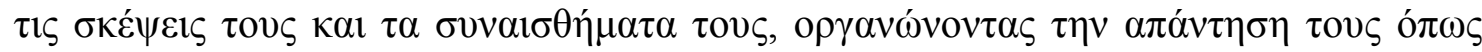

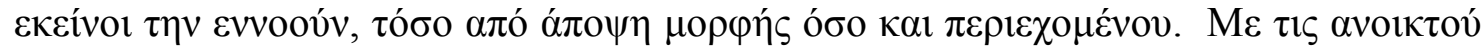

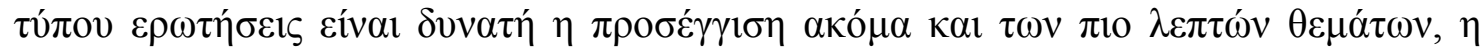

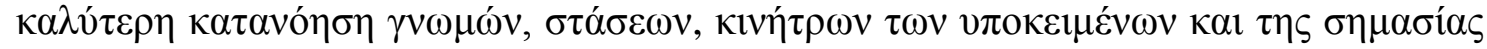

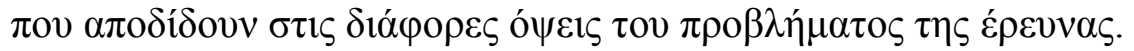

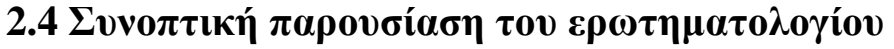

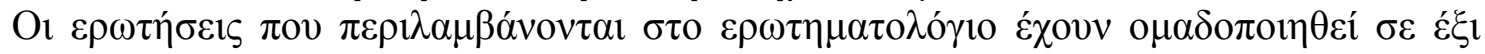

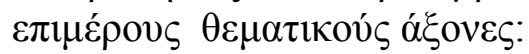

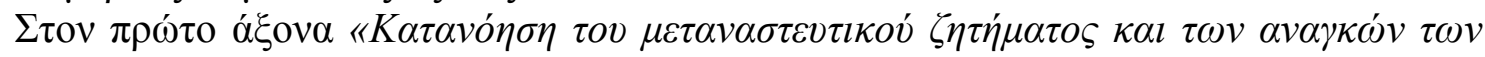

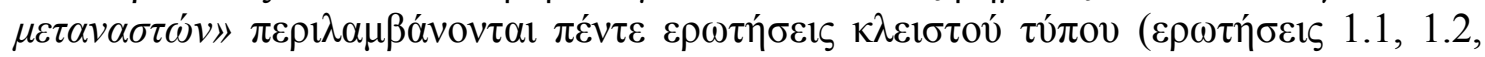

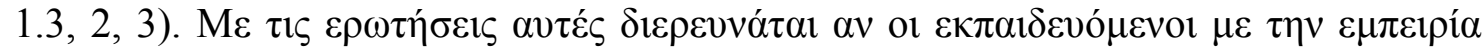

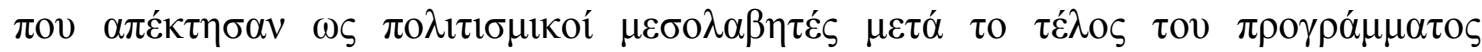

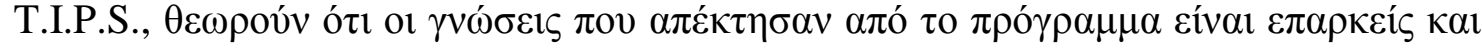
$\sigma \varepsilon \pi$ оเо $\beta \alpha \theta \mu$ ó.

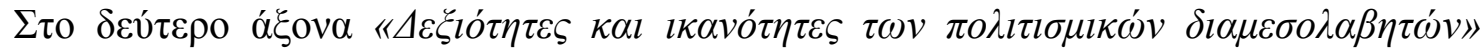

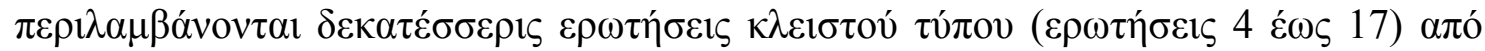

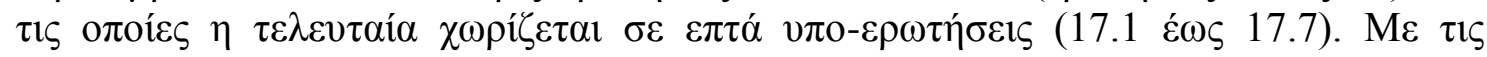

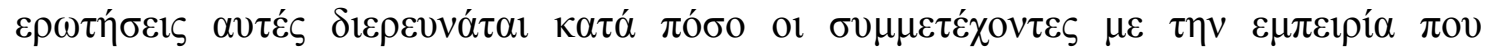

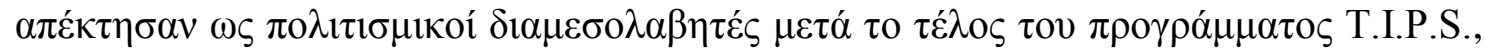

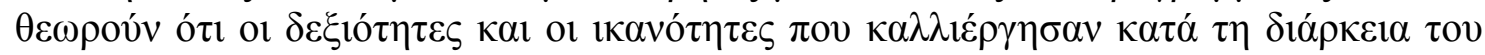

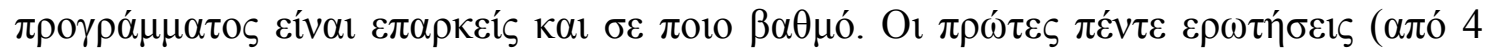

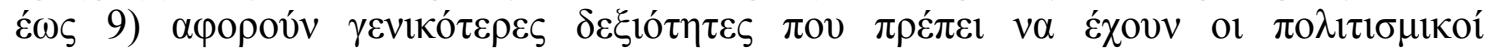

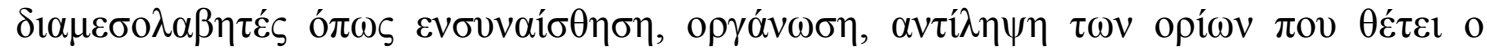




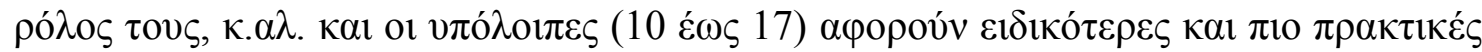

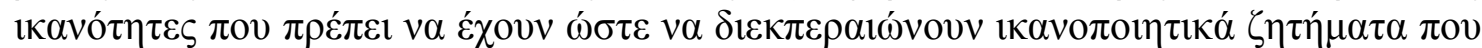

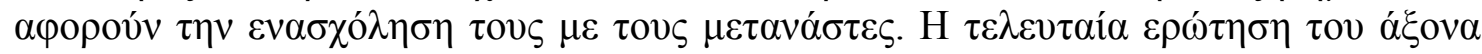

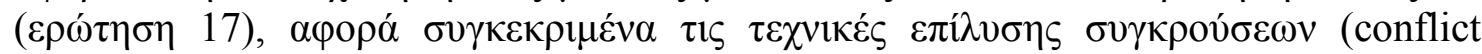

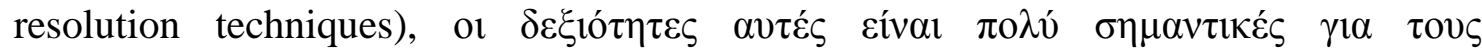

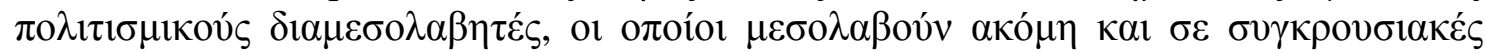
$\kappa \alpha \tau \alpha \sigma \tau \alpha ́ \sigma \varepsilon 1 \zeta$.

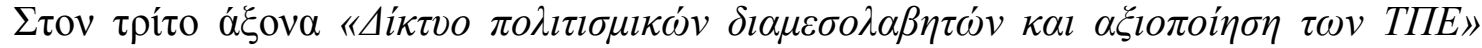

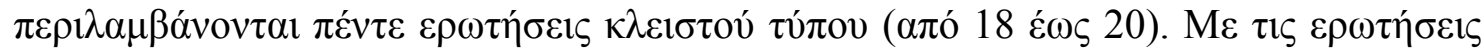

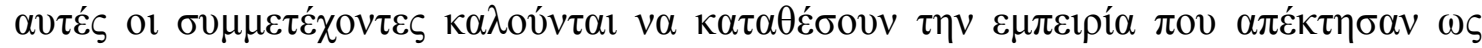

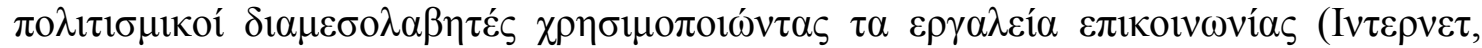

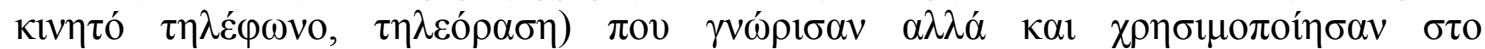

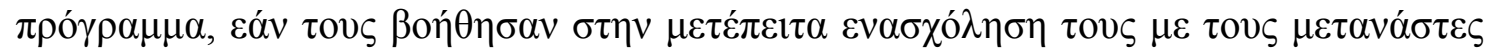

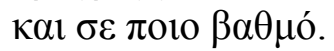

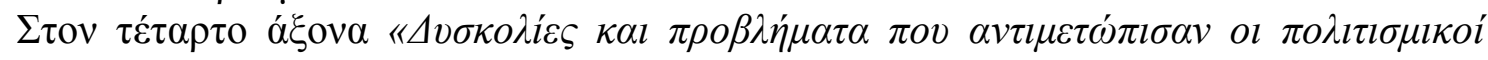

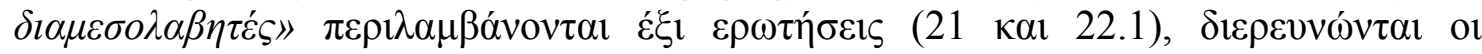

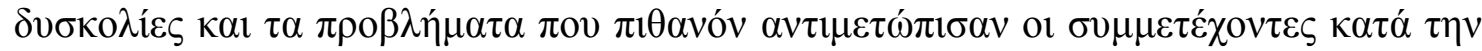

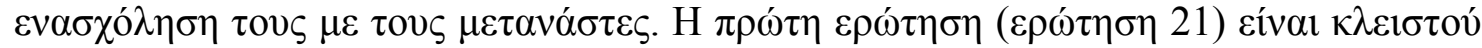

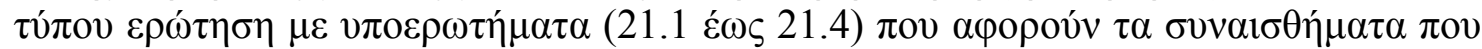

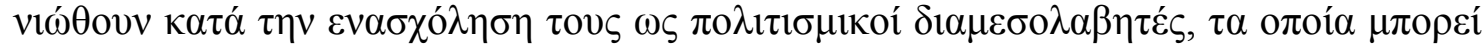

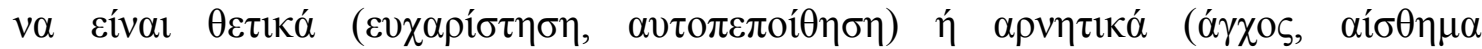

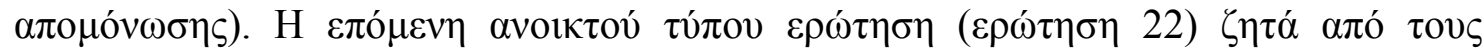

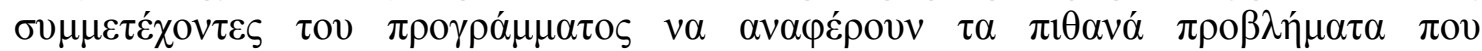

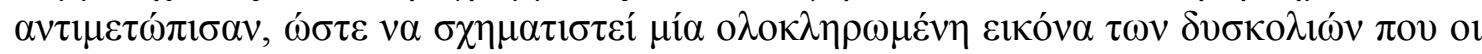

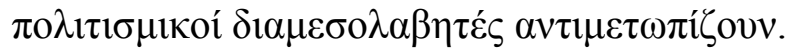

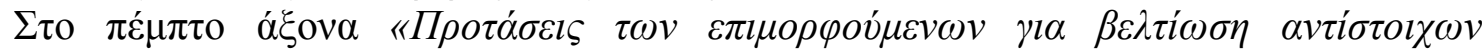

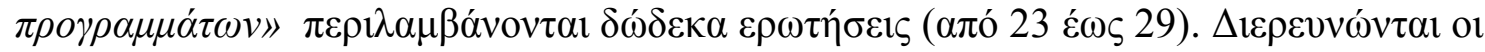

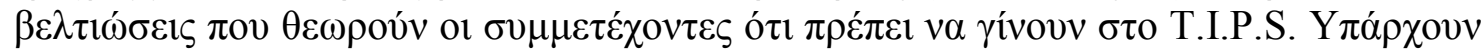

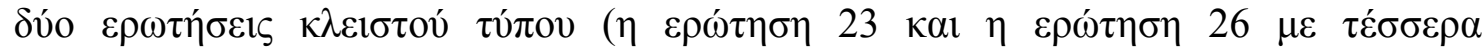

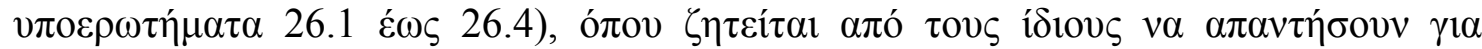

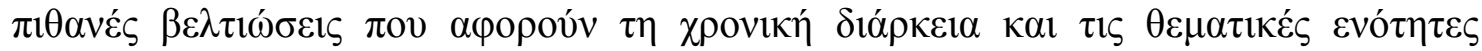

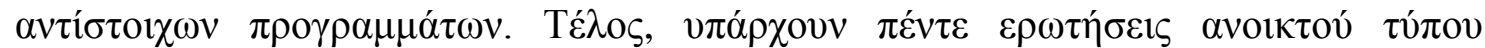

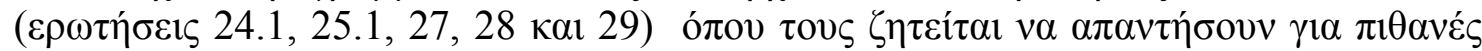

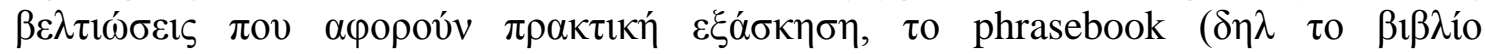

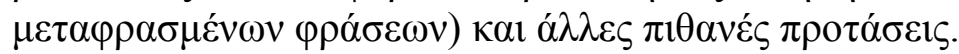

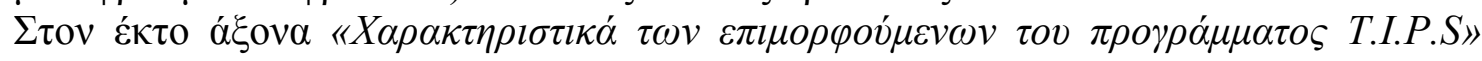

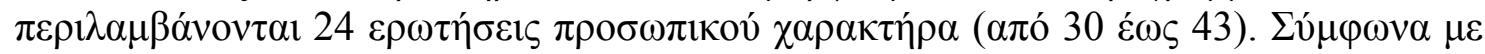

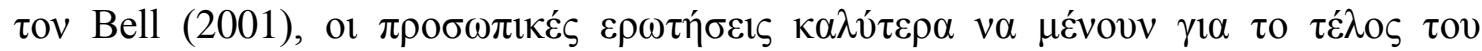

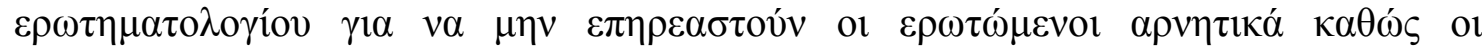

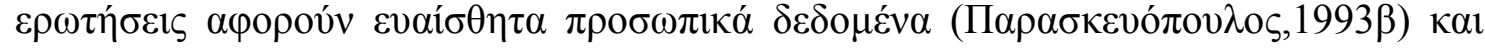

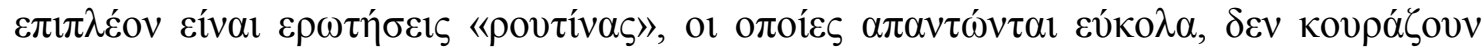

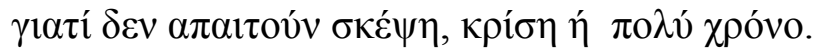

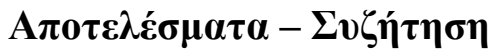

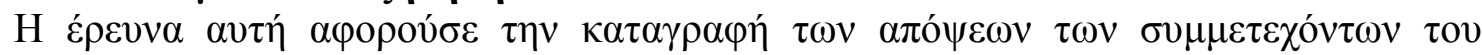

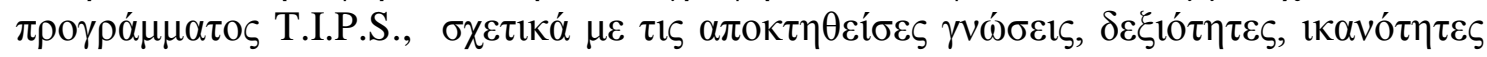

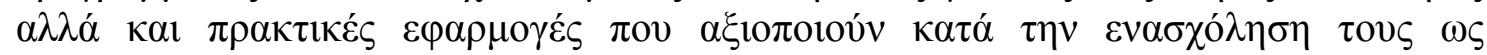

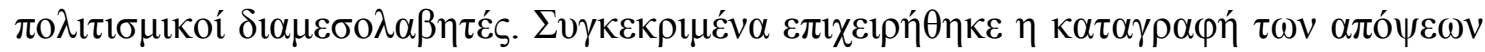

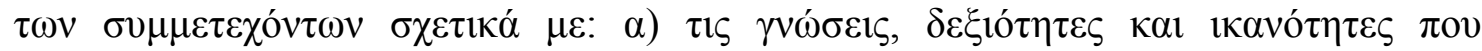

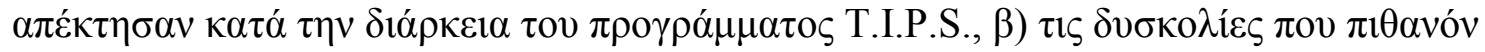




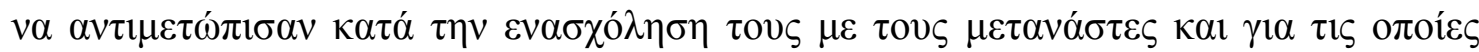

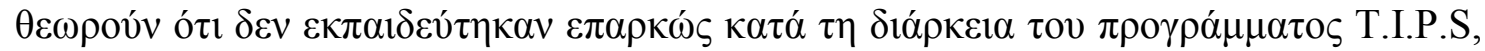

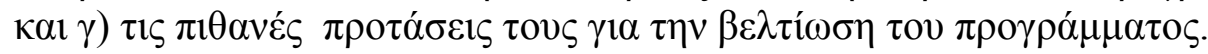

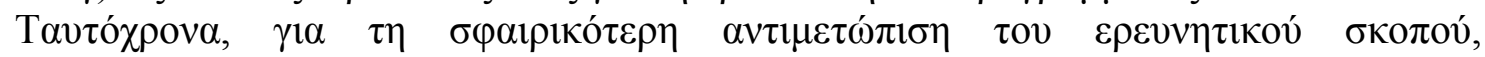

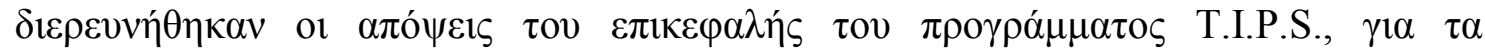

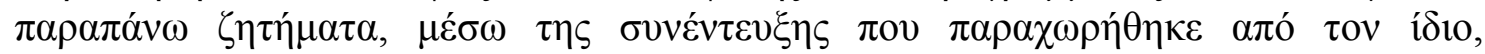

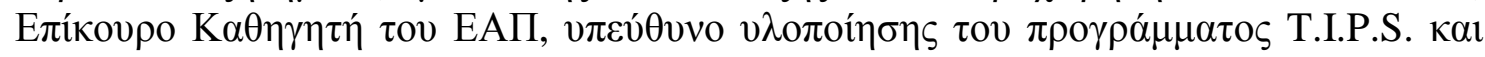

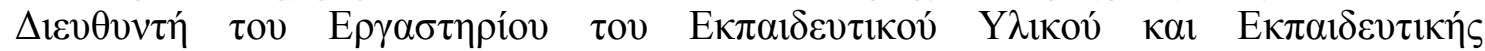

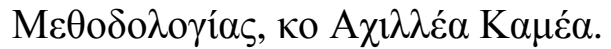

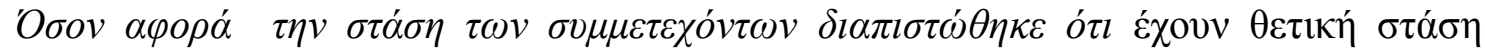

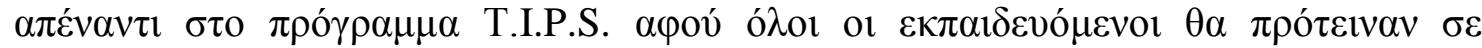

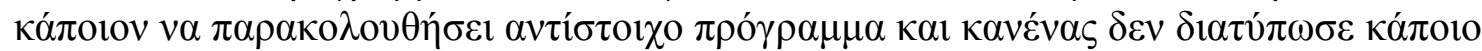

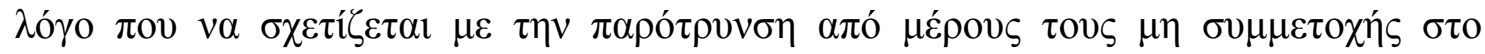

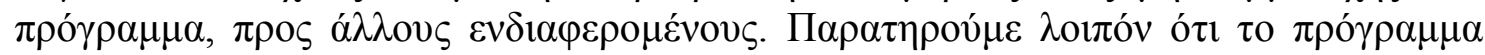

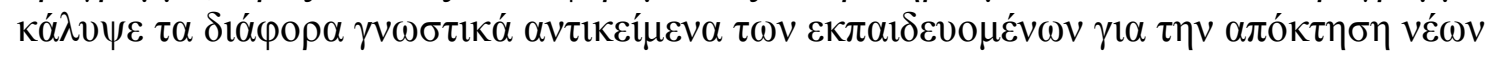

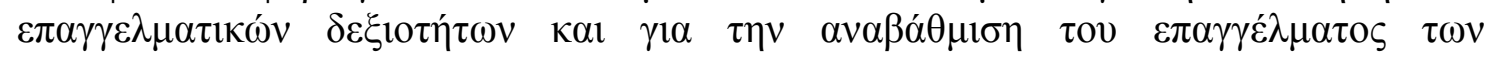

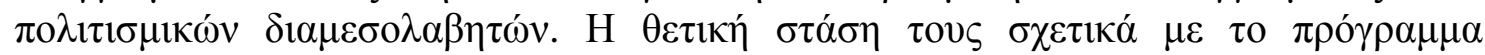

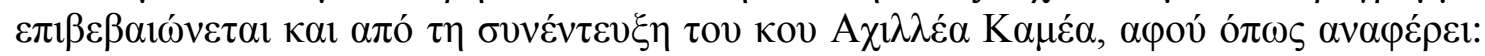

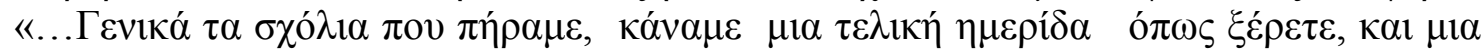

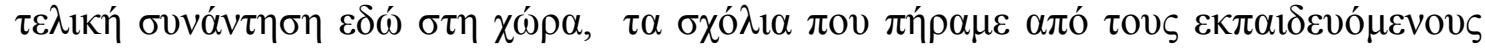

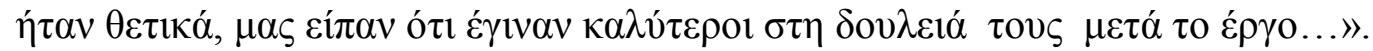

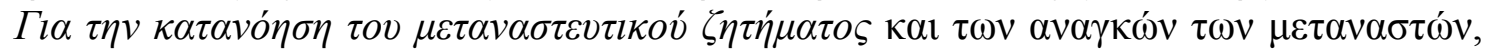

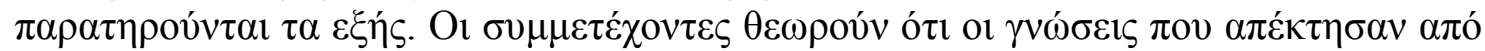

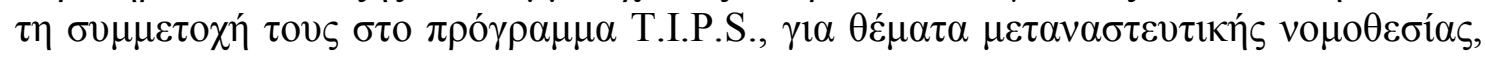

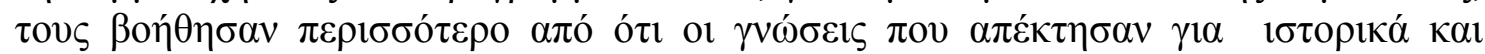

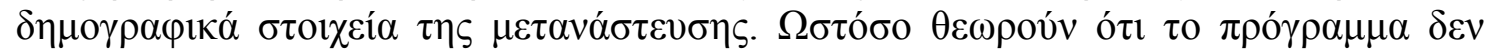

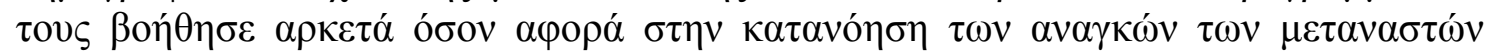

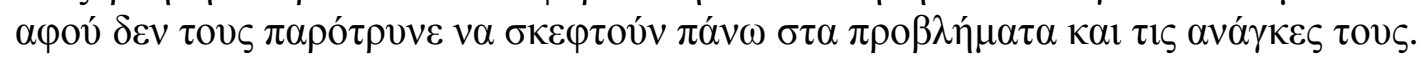

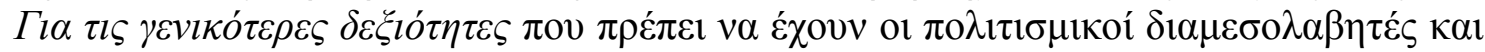

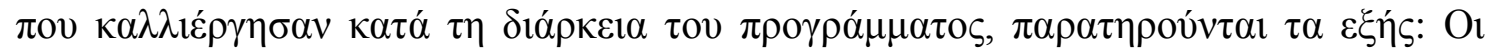

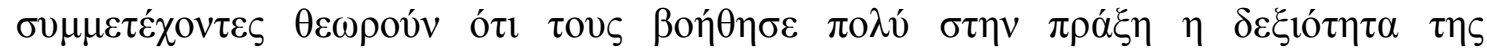

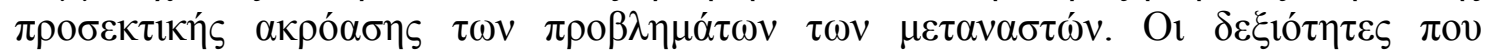

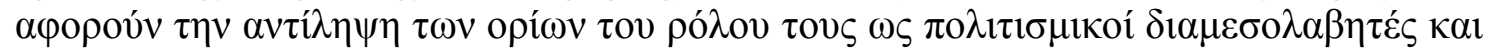

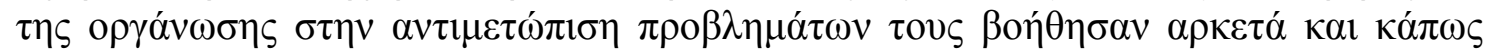

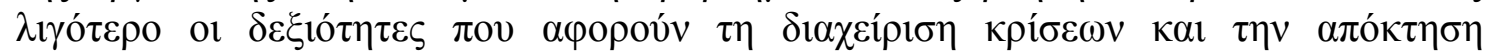

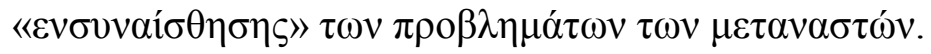

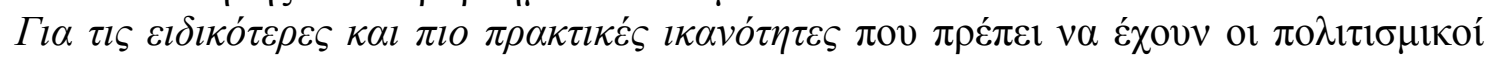

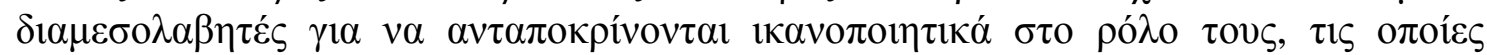

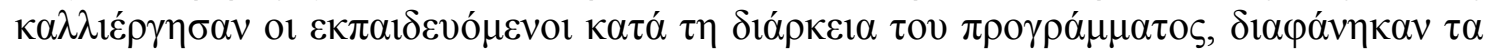

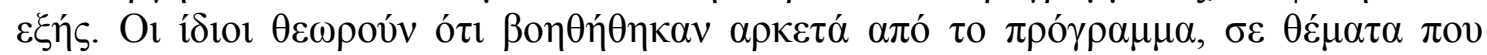

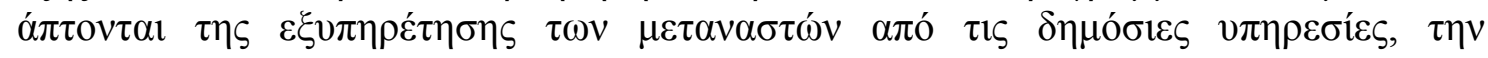

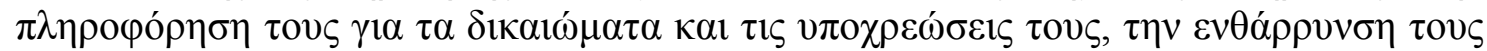

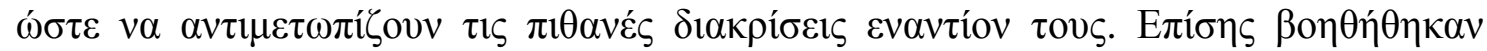

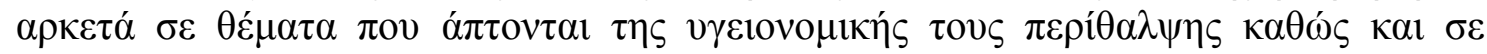

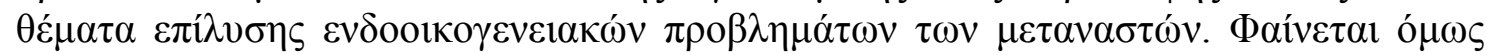

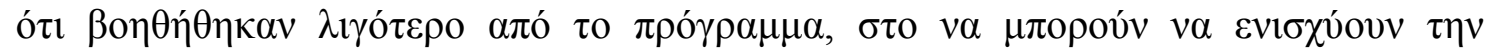

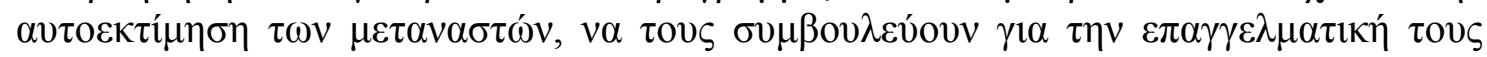

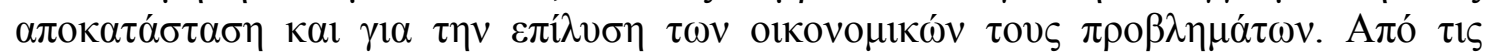

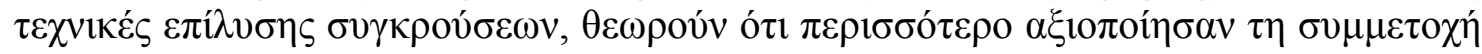

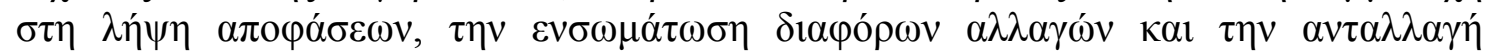




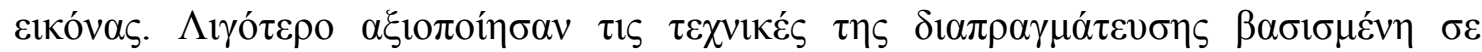

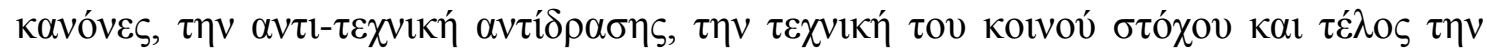

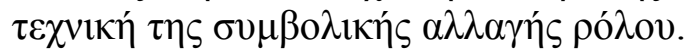

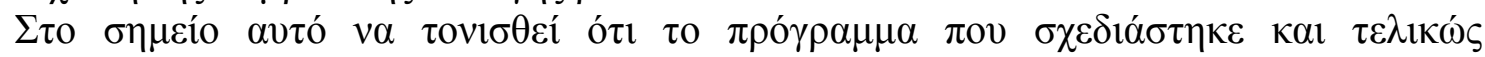

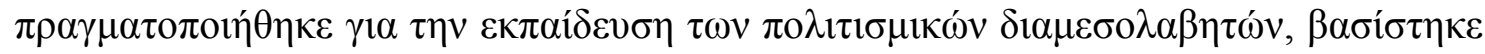

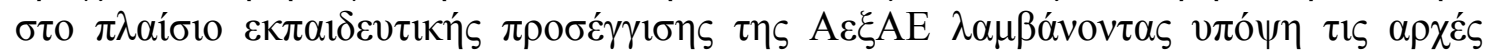

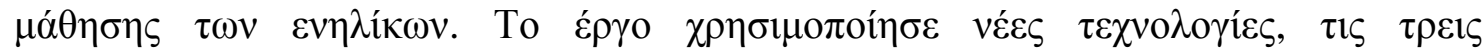

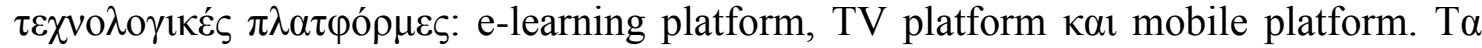

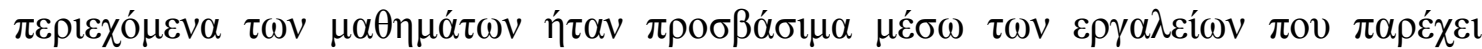

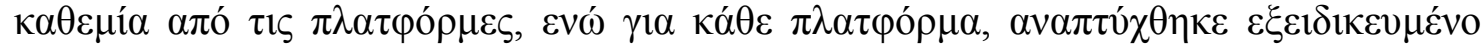
$\varepsilon \kappa \pi \alpha 1 \delta \varepsilon v \tau 1 \kappa o ́ ~ \pi \varepsilon \rho 1 \varepsilon \chi o ́ \mu \varepsilon v o$. H $\quad$

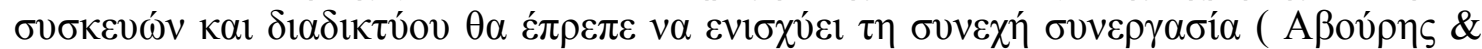

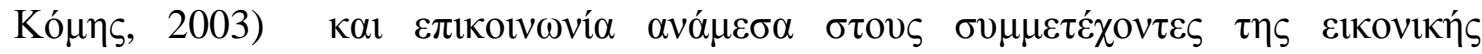

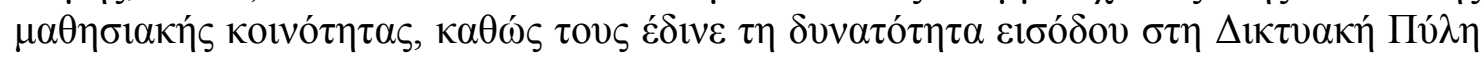

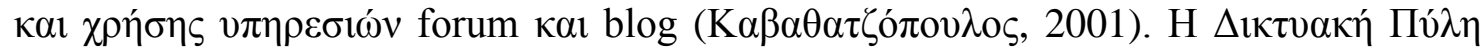

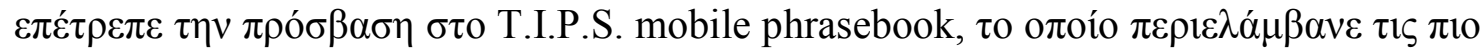

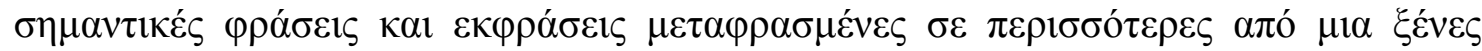

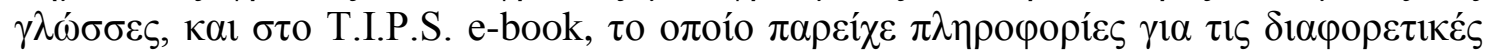

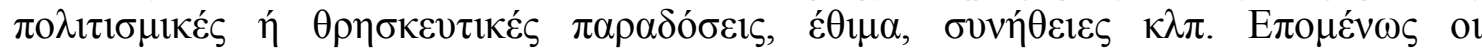

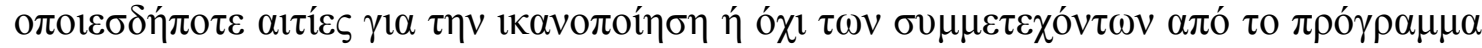

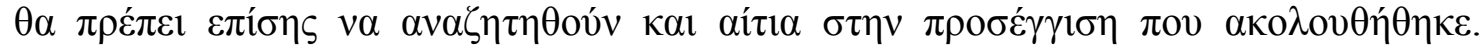

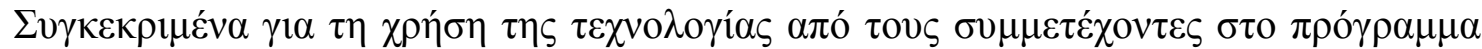

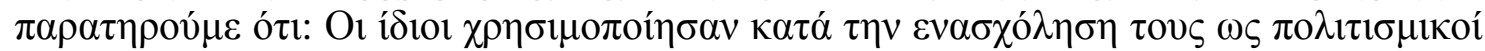

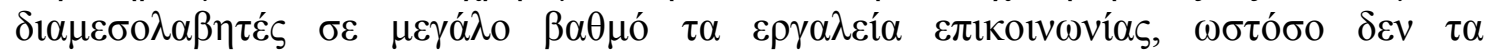

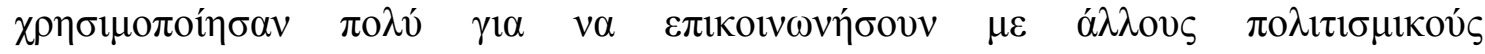

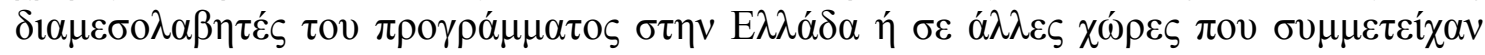

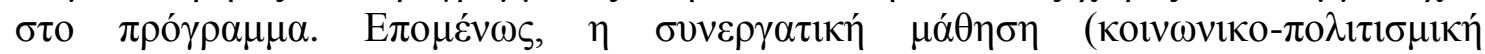

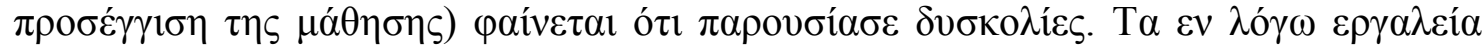

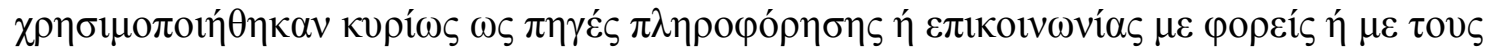

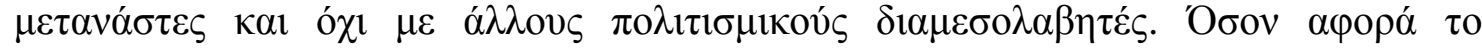

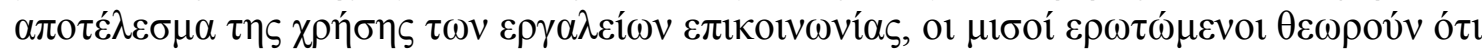

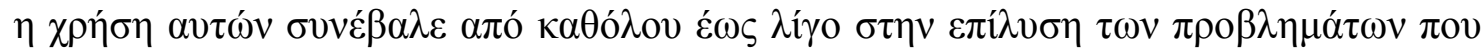

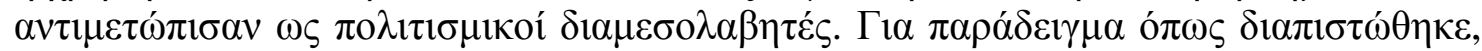

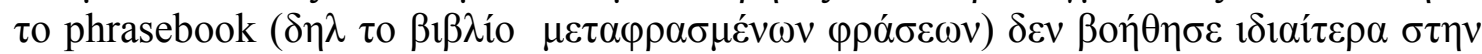

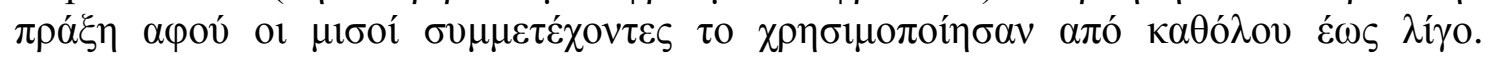

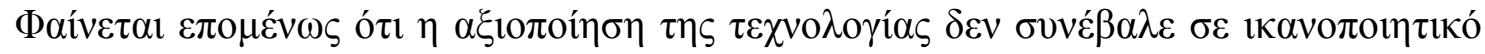

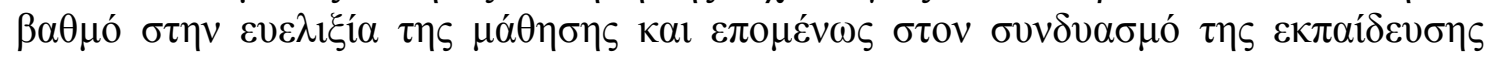

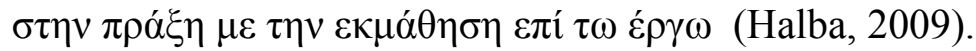

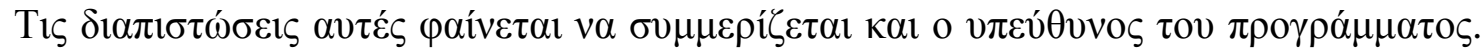

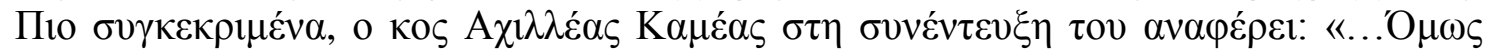

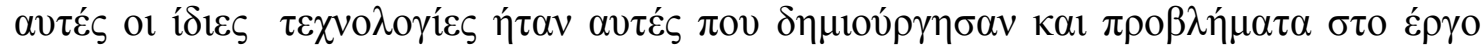

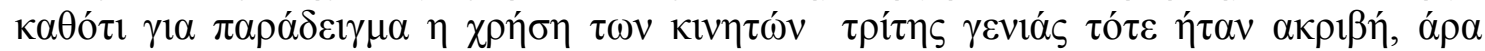

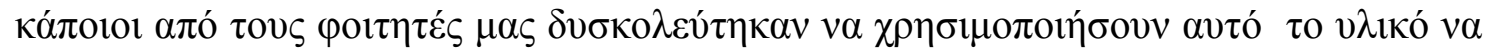

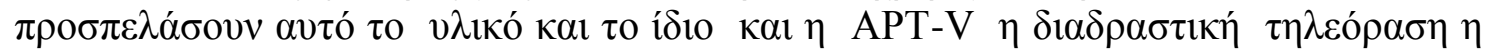

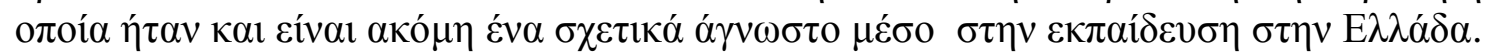

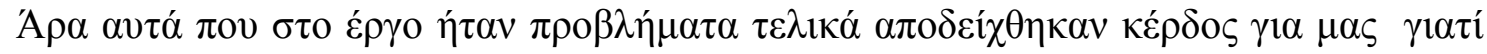
$\alpha v \alpha \gamma \kappa \alpha \sigma \tau \eta \dot{\kappa} \kappa \alpha \mu \varepsilon \alpha \tau \alpha \delta$ ov́ $\mu \varepsilon, v \alpha \tau \alpha \gamma \nu \omega \rho i ́ \sigma o v \mu \varepsilon, v \alpha \tau \alpha \lambda v^{\sigma} \sigma o v \mu \varepsilon \ldots »$.

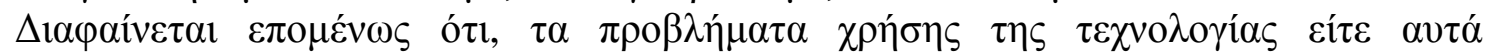

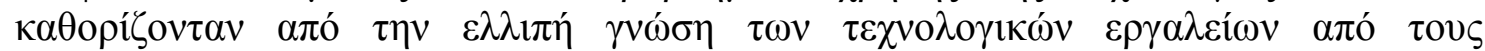

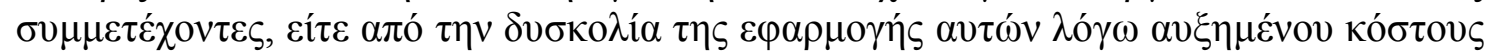




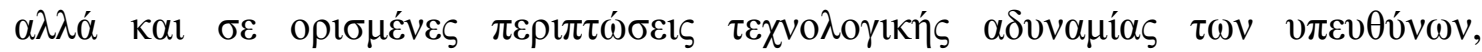

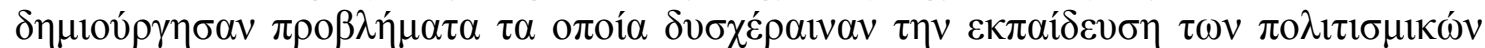

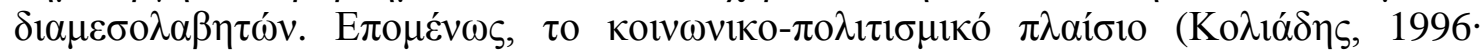

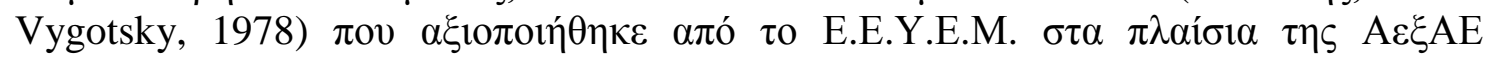

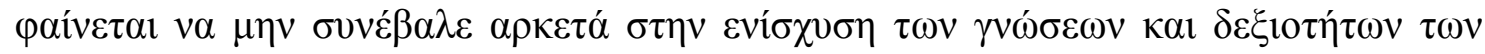

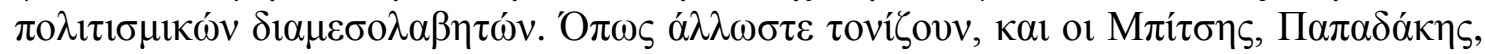

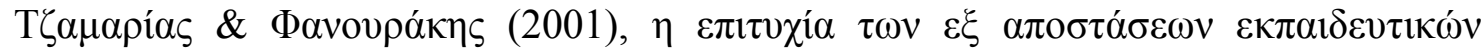

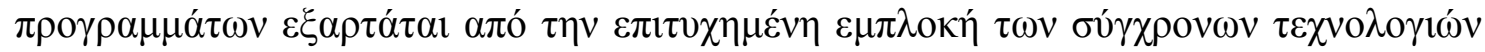

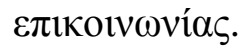

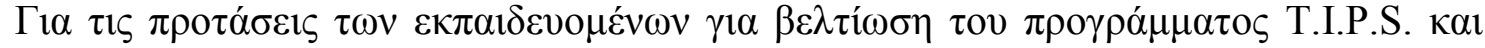

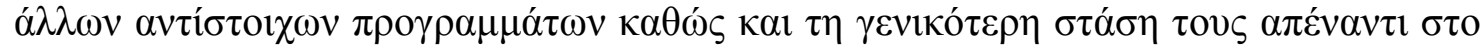

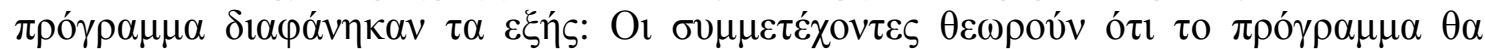

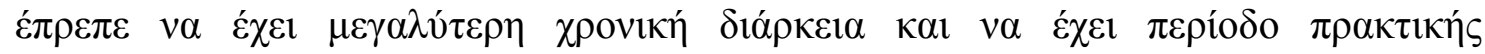

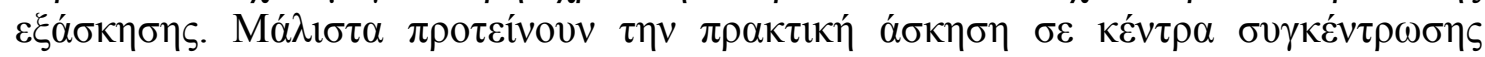

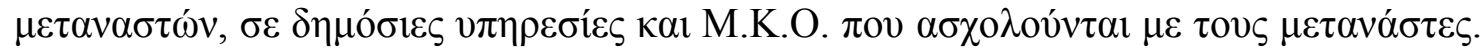

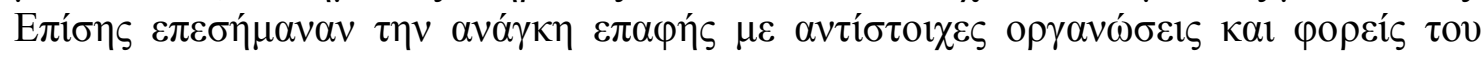
$\varepsilon \xi \omega \tau \varepsilon \rho \imath \kappa o v ́$.

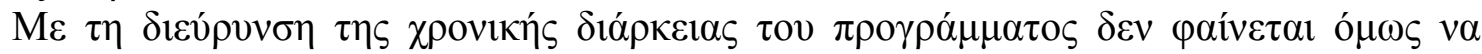

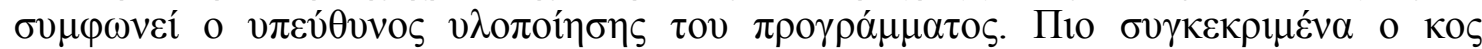

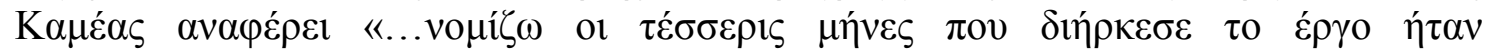

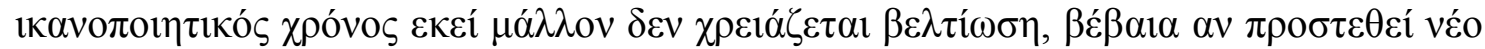

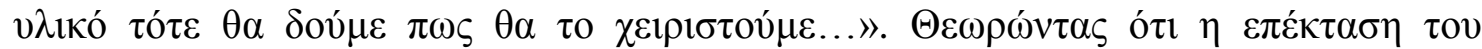

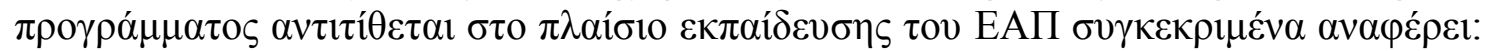

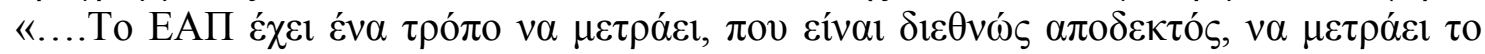

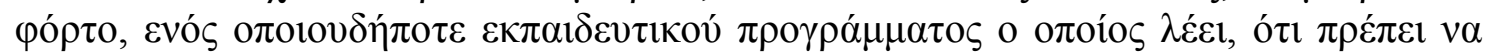

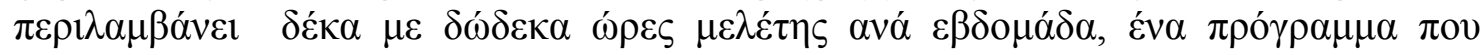

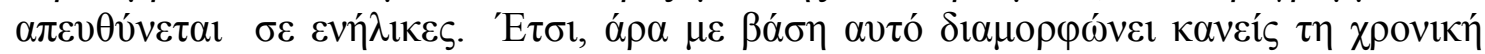

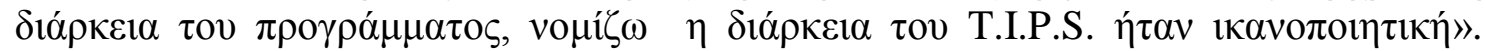

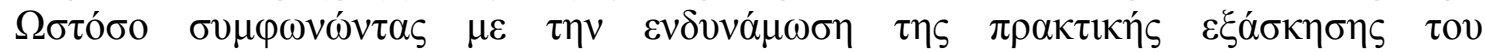

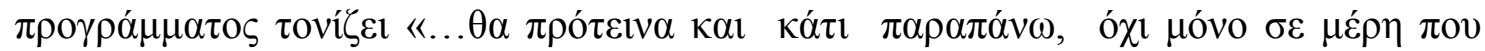

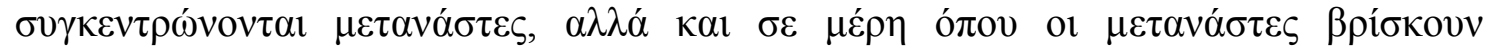

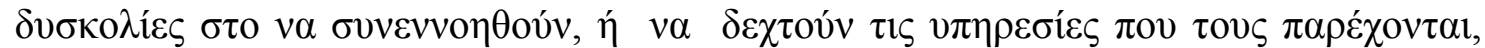

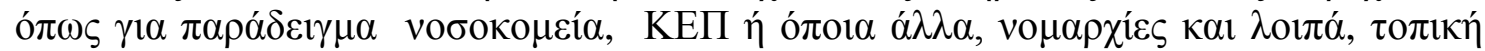

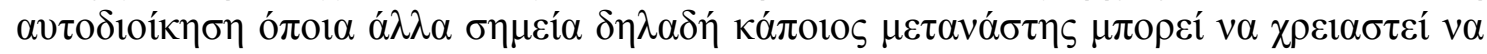

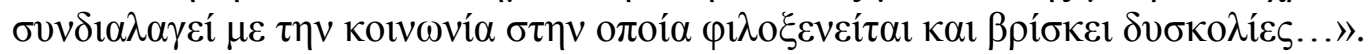

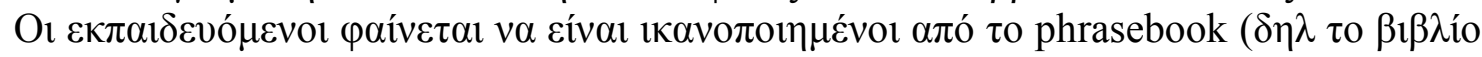

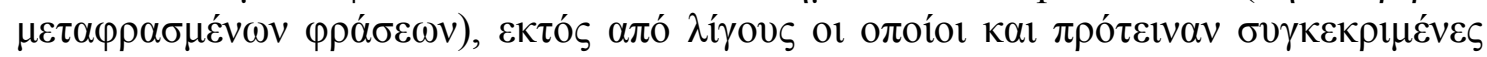

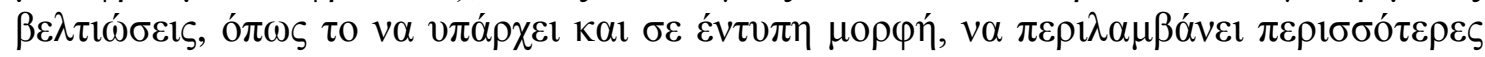

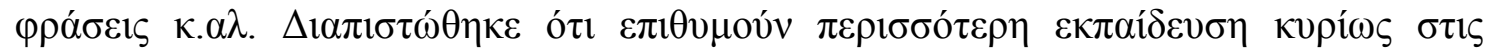

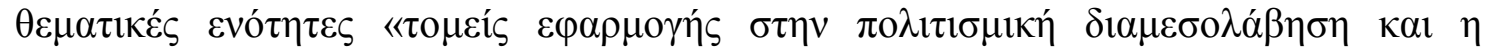

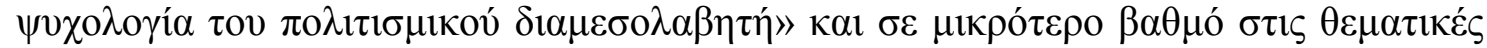

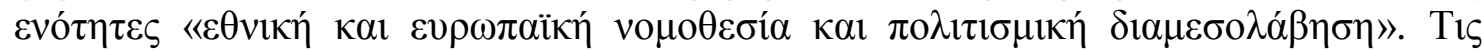

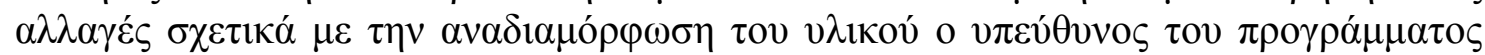

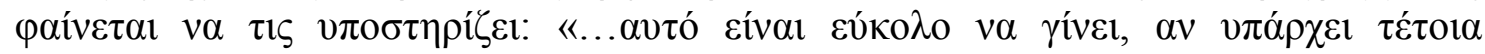

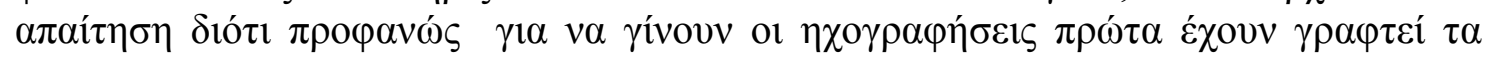

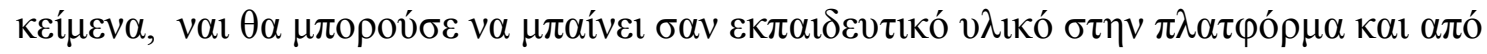

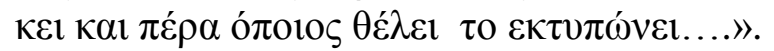

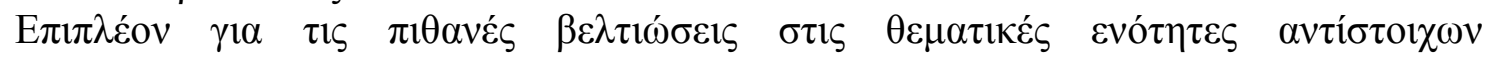

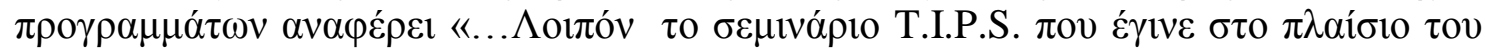

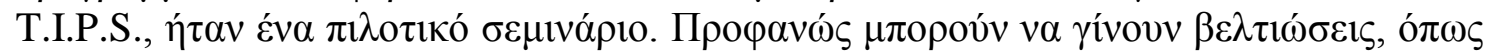




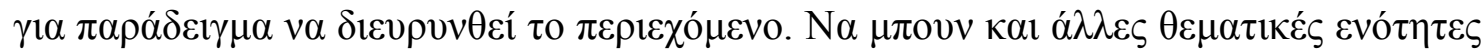

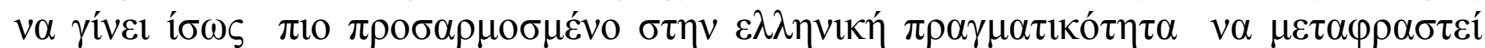

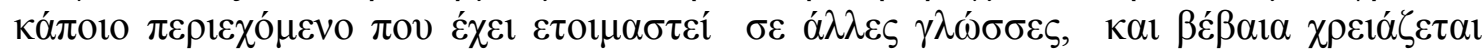

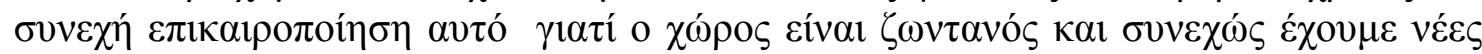

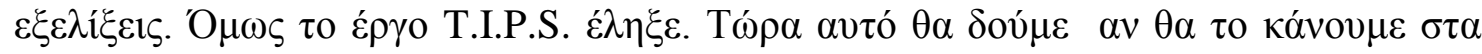

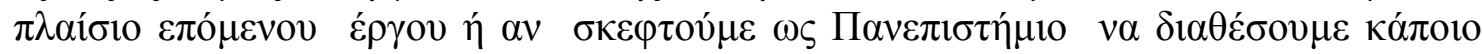

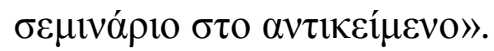

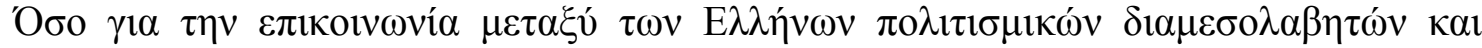

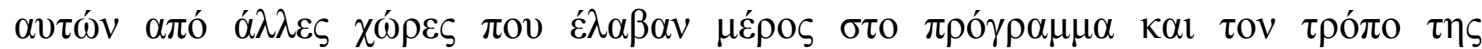

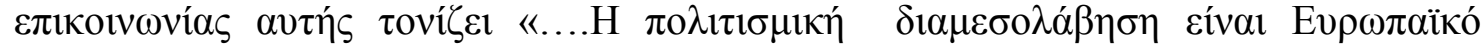

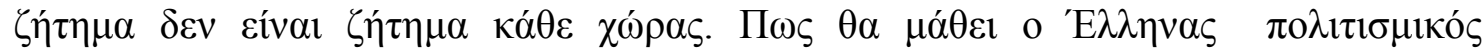

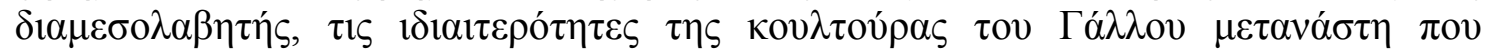

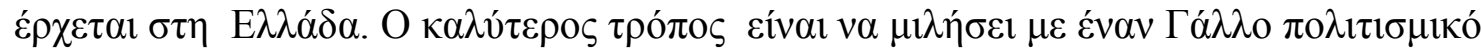

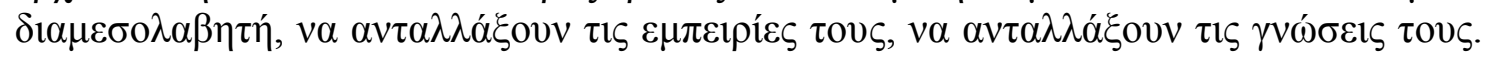

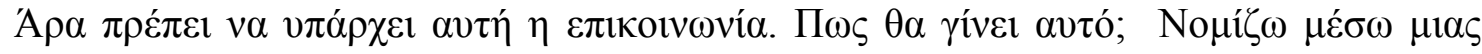

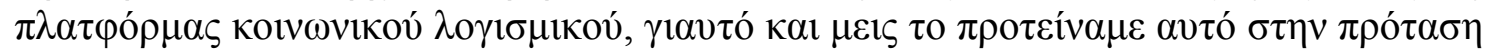

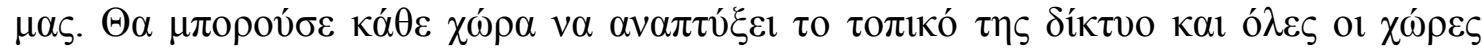

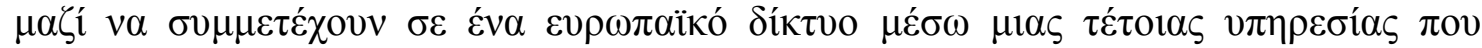

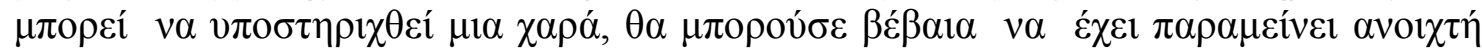

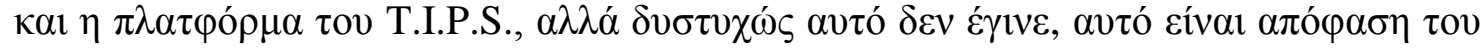

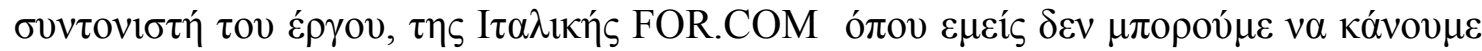
кá $\imath$...».

\section{$\Sigma v \mu \pi \varepsilon \rho \alpha ́ \sigma \mu \alpha \tau \alpha-\Pi \rho 0 \tau \alpha ́ \sigma \varepsilon ı \varsigma$}

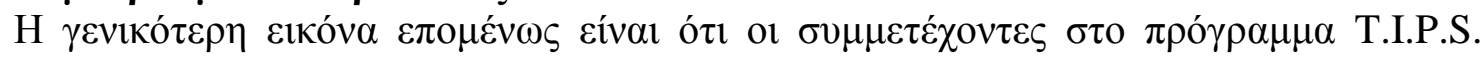

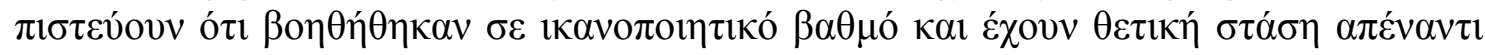

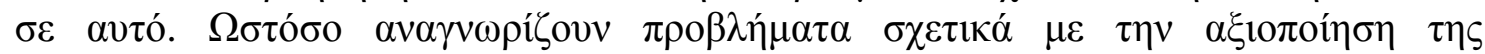

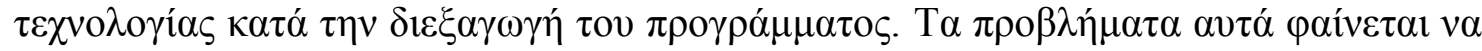

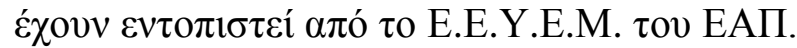

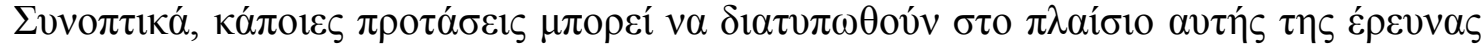

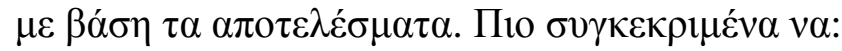

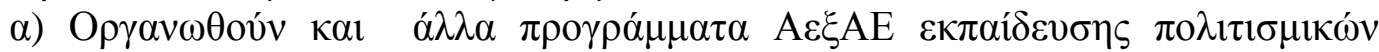

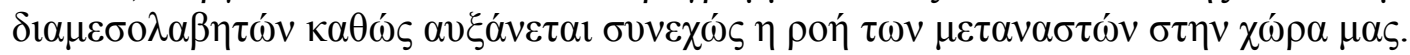

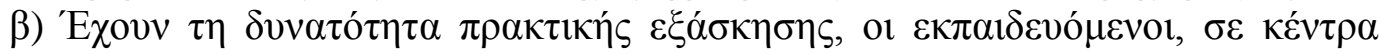

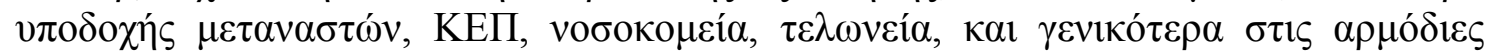

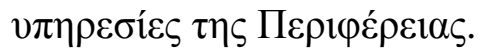

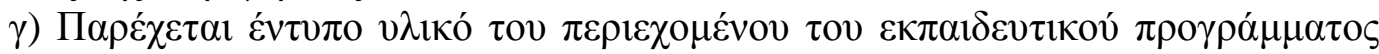

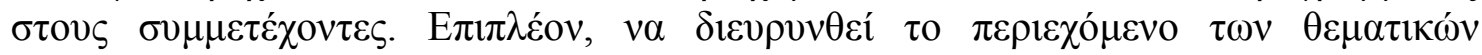

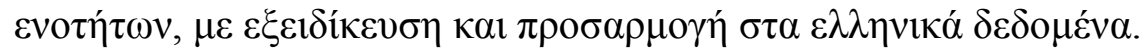

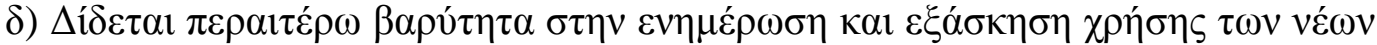

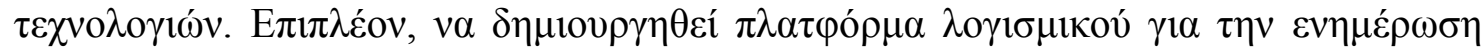

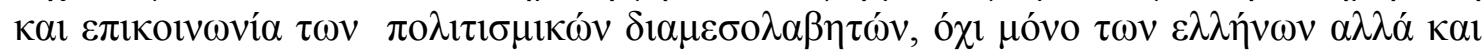

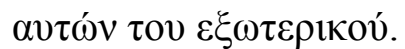

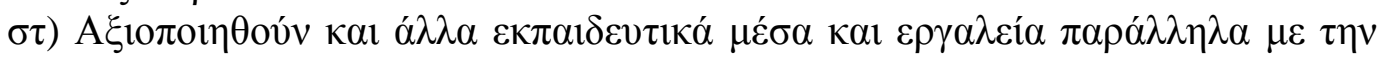
$\varepsilon \xi \alpha \pi 0 \sigma \tau \alpha \dot{\sigma} \sigma \omega \varsigma \varepsilon \kappa \pi \alpha i ́ \delta \varepsilon v \sigma \eta$ (Blended Learning).

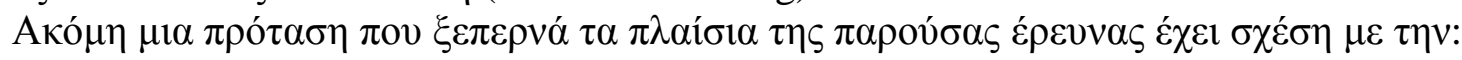

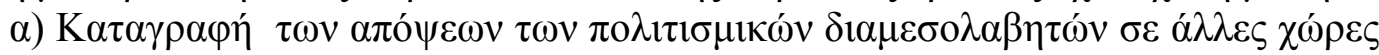

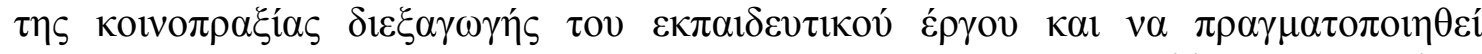

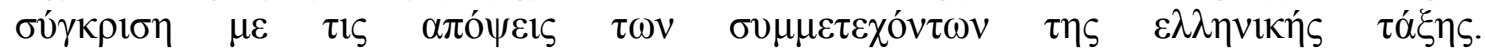




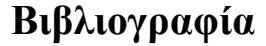

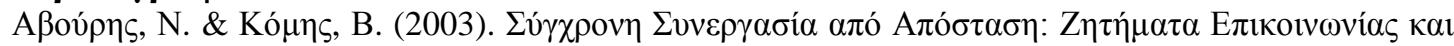

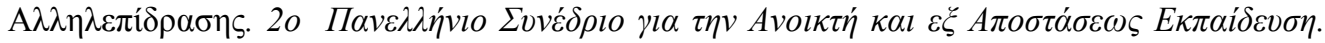

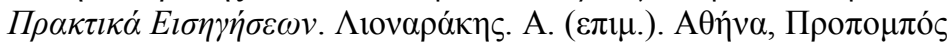

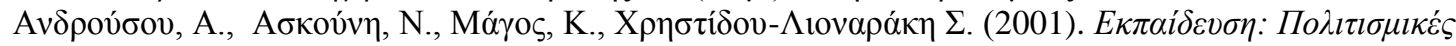

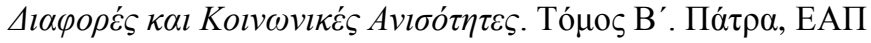

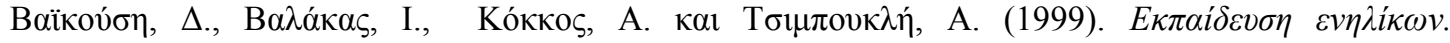

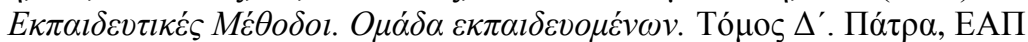

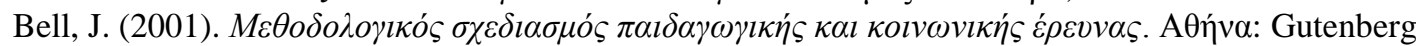

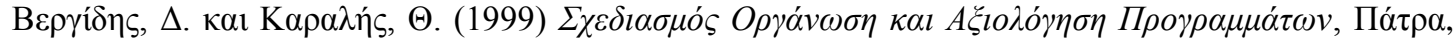

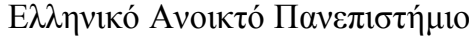

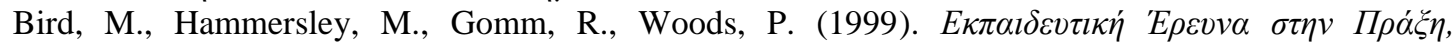

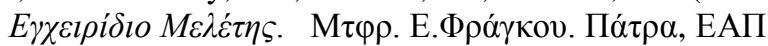

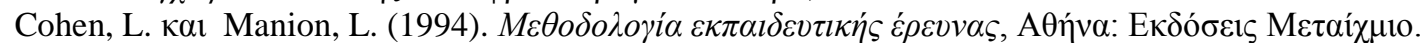

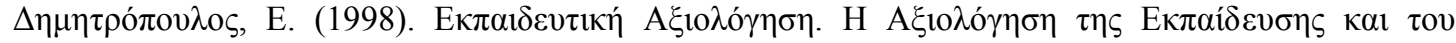

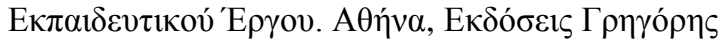

Halba, B. (2009). T.I.P.S. For intercultural dialogue-T-learning to Improve Professionall Skills For Intercultural dialogue. Paris ,October7th Renovabis international conference "Migration in Europe_Change or danger?"'Analyses and concepts(freising,28-30.08.2003).Speech by Walter Schwimmer,secretory General of the Council of Europe)

Halba, B. (2008). TIPS , Rapports pour la France, Leonardo da Vinci project, initiated by For.Com (Italy). May 2008

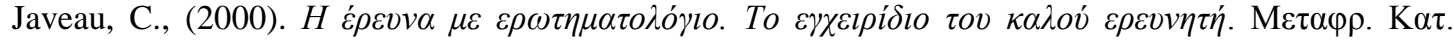

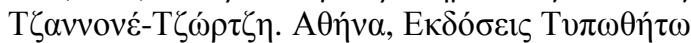

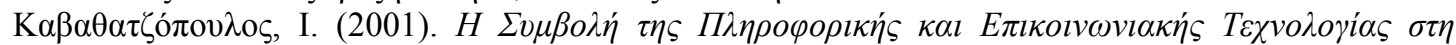

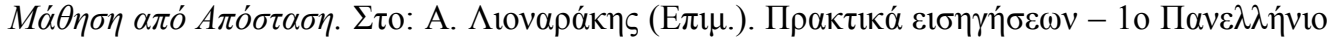

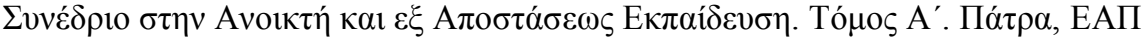

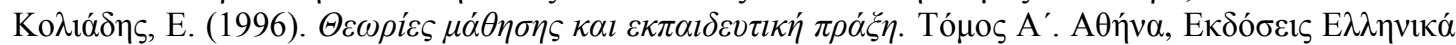
$\Gamma \rho \alpha ́ \mu \mu \alpha \tau \alpha$

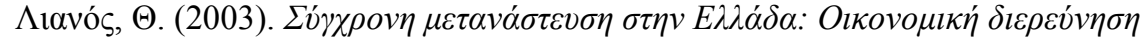

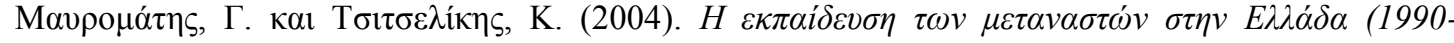

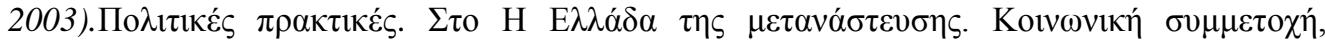

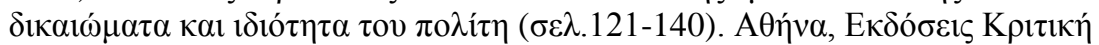

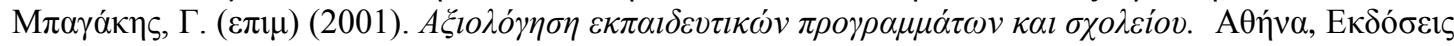

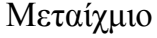

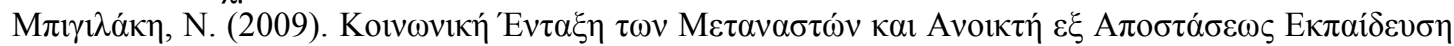
$\sigma \tau \eta \nu$ E $\lambda \lambda \alpha \dot{\delta} \alpha$. 5th International Conference in Open \& Distance Learning - November 2009, Athens

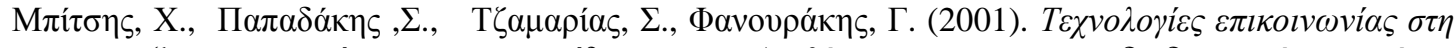

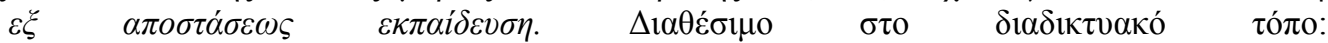
http://www.eap.gr/news/exaggeliasynedriou/synedrio/html/sect6/150.htm

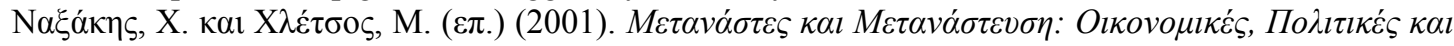

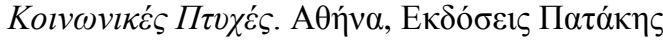

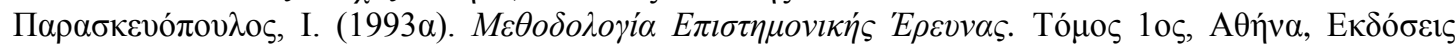

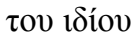

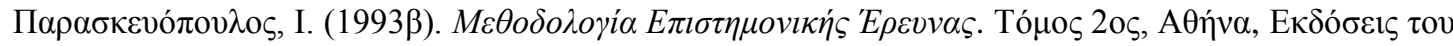
ió́ov

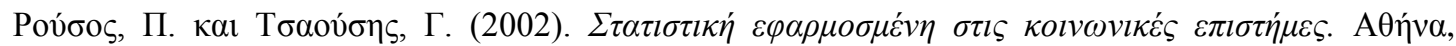

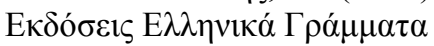

Silverman, D. (2000). Analyzing Talk and Text. In N. K. Denzin, \& Y. S. Lincoln (Eds), Handbook of Qualitative Research, $2 * E d$. London: Sage

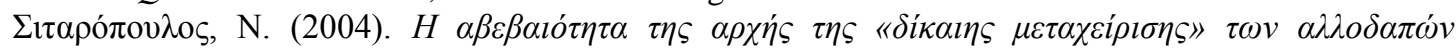

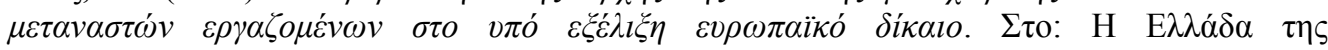

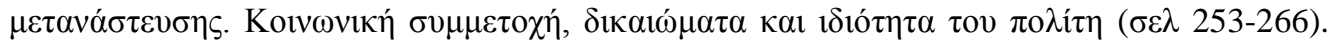

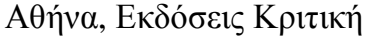

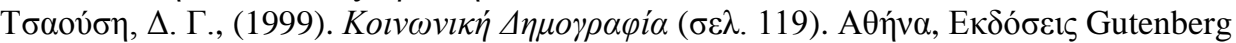

Triantafyllidou, A. Gropas R. (2007). European Immigration: a sourcebook. Hampshire. Ashgate.

Vygotsky, L .S., (1978). Mind in Society: the development of higher psychological processes. MA: Harvard University Prees, Cambridge 
$6^{\text {th }}$ International Conference in Open \& Distance Learning - November 2011, Loutraki, Greece - PROCEEDINGS

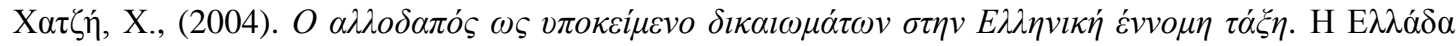

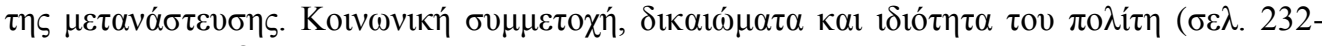

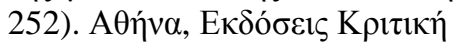

